\title{
Liposomal Encapsulated Curcumin Effectively Attenuates Neuroinflammatory and Reactive Astrogliosis Reactions in Glia Cells and Organotypic Brain Slices
}

This article was published in the following Dove Press journal:

International Journal of Nanomedicine

\author{
Christina Schmitt (D) \\ Anna Lechanteur ${ }^{2}$ \\ François Cossais' \\ Coralie Bellefroid $^{2}$ \\ Philipp Arnold (D) \\ Ralph Lucius' \\ Janka Held-Feindt ${ }^{3}$ \\ Geraldine Piel $^{2}$ \\ Kirsten Hattermann' \\ 'Institute of Anatomy, University Kiel, \\ Kiel D-24098, Germany; ${ }^{2}$ Laboratory of \\ Pharmaceutical Technology and \\ Biopharmacy (LTPB), CIRM, University of \\ Liège, Liège, Belgium; ${ }^{3}$ Department of \\ Neurosurgery, University Medical Center \\ Schleswig-Holstein UKSH, Kiel D-24I05, \\ Germany
}

\begin{abstract}
Introduction: The polyphenolic spice and food coloring ingredient curcumin has beneficial effects in a broad variety of inflammatory diseases. Amongst them, curcumin has been shown to attenuate microglia reaction and prevent from glial scar formation in spinal cord and brain injuries.
\end{abstract}

Methods: We developed a protocol for the efficient encapsulation of curcumin as a model for anti-inflammatory drugs yielding long-term stable, non-toxic liposomes with favorable physicochemical properties. Subsequently, we evaluate the effects of liposomal curcumin in experimental models for neuroinflammation and reactive astrogliosis.

Results: We could show that liposomal curcumin can efficiently reduce the reactivity of human microglia and astrocytes and preserve tissue integrity of murine organotypic cortex slices.

Discussion and Perspective: In perspective, we want to administer this curcumin formulation in brain implant coatings to prevent neuroinflammation and glial scar formation as foreign body responses of the brain towards implanted materials.

Keywords: glial scar, neuroinflammation, nanoparticles, brain implants

\section{Introduction}

Curcumin is a natural polyphenolic compound that can be obtained from the rhizome of turmeric, Curcuma longa, and is widely used as a spice and food coloring ingredient, but also in Ayurvedic medicine. ${ }^{1}$ Apart from its use as a food additive, beneficial effects of curcumin, its derivatives and analogues have been investigated in vitro and in vivo up to clinical trials in a broad variety of diseases, for example, in cancer (eg breast, colorectal, pancreatic cancer) and inflammatory diseases [eg inflammatory bowel disease, osteoarthritis and rheumatoid arthritis. ${ }^{2-7}$ In the nervous system, the effects of curcumin and its derivatives have been studied in the context of neurodegenerative diseases, eg Alzheimer's and Parkinson's disease, pathological pain, epilepsy and cerebral ischemia. ${ }^{8-16}$ These and other studies revealed that curcumin may influence several intracellular signaling cascades, yielding neuroprotective and anti-inflammatory microglia-attenuating effects. ${ }^{17-25}$ In recent studies, curcumin has also been described to prevent glial scar formation in spinal cord and brain injuries. ${ }^{26-29}$ In experimental studies on
Correspondence: Kirsten Hattermann Institute of Anatomy, University Kiel, Olshausenstr. 40, Kiel D-24098, Germany $\mathrm{Tel}+494318802460$

Fax +494318801557

Email k.hattermann@anat.uni-kiel.de 
spinal cord injuries, curcumin could reduce in vitro and in vivo the production of pro-inflammatory mediators, the reactive expression of gliosis/fibrosis markers and the deposition of chondroitin sulfate proteoglycans, presumably by modulating several, partly diverging signaling pathways. ${ }^{27-30}$ However, curcumin is quite instable in aqueous solutions, sensitive to light and hardly bioavailable in experimental studies. ${ }^{31-33}$ These facts and the failure of some recent clinical trials paved the way for the development of better bioavailable and more stable curcumin formulations, for example, by the use of micro-emulsions, nanofibers or nanoparticles. ${ }^{5,8,34-36}$ In particular, drug-loaded nanoparticles are known for years to be good carriers for the encapsulation, transportation and release of drugs with tailorable release kinetics of the pharmaceutical compound to the target side as well as enhanced solubility and stability of most drugs, resulting in improved pharmacokinetic profiles. ${ }^{37-39}$ Liposomal formulations have successfully climbed the step from an experimental concept to clinical application as an FDA approved nano-carrier for a variety of drugs, and liposomal encapsulation has also been shown to be a promising formulation strategy for curcumin, due to its biocompatibility and biodegradable properties. ${ }^{40,41}$ Liposomes are artificial membrane-like bilayer structures, consisting of cholesterol and other non-toxic phospholipids, that are formed by exposing the lipid headgroups to a highly hydrophilic aqueous surrounding, allowing for the encapsulation of hydrophilic as well as hydrophobic drugs, such as curcumin. ${ }^{42-44}$ Several reports show beneficial effects of liposomal curcumin formulations, for example, on growth-inhibition and pro-apoptotic effects on cancer cells, higher anti-inflammative and -oxidative effects in vaginal inflammation models, increased bioavailability upon oral administration and improved skin-penetration in melanoma treatment. ${ }^{45-49}$ However, only little has been shown yet for liposomal curcumin applications in the brain. To date, nano-formulated curcumin has been shown to attenuate microglia activation and to protect the blood-brain barrier integrity, but more comprehensive studies on the use of liposomal encapsulated curcumin to prevent glial scar formation are still missing. ${ }^{50,51}$

The aim of this study was to develop a liposomal nanocarrier formulation and to evaluate its protective effect in experimental microglial inflammation as well as reactive astrogliosis processes. The idea behind this concept is that, in local brain therapy strategies, the glial scaring process evoked by the brain implant materials and impairing the implants function (eg drug delivery or signal recording) could in perspective be reduced by the (co-) administration of an anti-inflammatory drug such as curcumin. ${ }^{52,53}$ The formulation of curcumin (or other anti-inflammatory drugs) in long-term stable, non-toxic nano-carriers would facilitate the local deposition, eg in biodegradable implant coatings. Thus, we developed a protocol for the liposomal encapsulation of curcumin (we termed these liposomes LipoCur) and evaluated their physicochemical properties, long-term stability and in vitro drug release kinetic. As microglia promoted neuroinflammation and astrocyte mediated reactive gliosis mainly contribute to the brain foreign body response (compare Figure 1A) we focused on our first experimental approaches on these cell types. Using human microglia and astrocytes, we analyzed the effects of liposomal curcumin in comparison to soluble "free" curcumin and empty liposomes on cell viability. To experimentally mimic the foreign body response in vitro, we challenged human microglia with lipopolysaccharide (LPS) to induce neuroinflammation (Figure 1B) and stimulated human astrocytes with a cytokine cocktail to induce reactive gliosis (Figure 1C). In these experimental settings, we analyzed the effect of liposomal curcumin (and for comparison "free" soluble curcumin and empty liposomes) on the expression of pro-inflammatory markers in microglial cells, and on the expression of glial scarring associated genes in cytokine-activated astrocytes. Additionally, we tested the effects of liposomal curcumin on LPS-challenged acute brain slices obtained from the mouse cortex to verify our observations in the complex tissue composition of the murine brain (Figure 1D).

\section{Materials and Methods}

\section{Preparation of Liposomal Nano-Carriers}

Briefly, lipids and curcumin were dissolved in $3 \mathrm{~mL}$ absolute ethanol at a total concentration of $1.34 \mathrm{mg} / \mathrm{mL}$ dipalmitoylphosphatidylcholine (DPPC, 16:0 PC, Avanti Polar Lipids, Alabaster, AL), $0.26 \mathrm{mg} / \mathrm{mL}$ cholesterol (SigmaAldrich/Merck, Darmstadt, Germany) and $0.063 \mathrm{mg} / \mathrm{mL}$ curcumin (Sigma-Aldrich) respectively, and mixed by slightly shaking of a $100 \mathrm{~mL}$ round bottom flask. The organic solvent was then removed using a rotary evaporator (Buchi R200, Flawil, Switzerland) for $1 \mathrm{~h}$ at $37^{\circ} \mathrm{C}$. The lipid film was rehydrated with $2 \mathrm{~mL}$ ultrapure water, vortexed and directly extruded through a polycarbonate membrane $\left(200 \mathrm{~nm}\right.$ and $100 \mathrm{~nm}$, Whatman ${ }^{\circledR}$, Fisher Scientific, Merelbeke, Belgium) at $51^{\circ} \mathrm{C}$. Thereafter, 
A

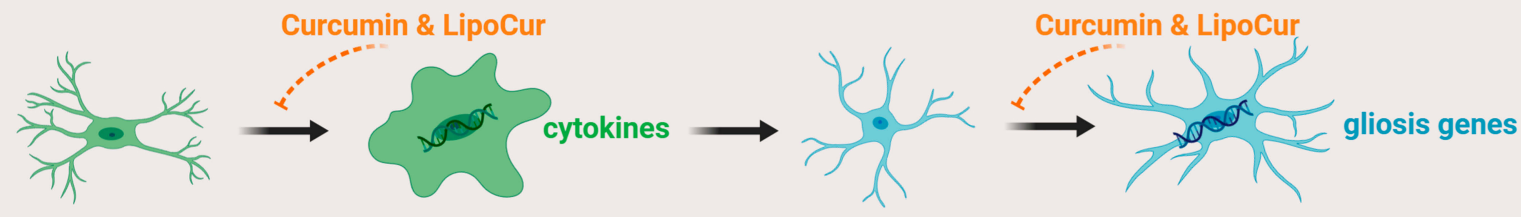

microglia activation

astrocytes activation

B

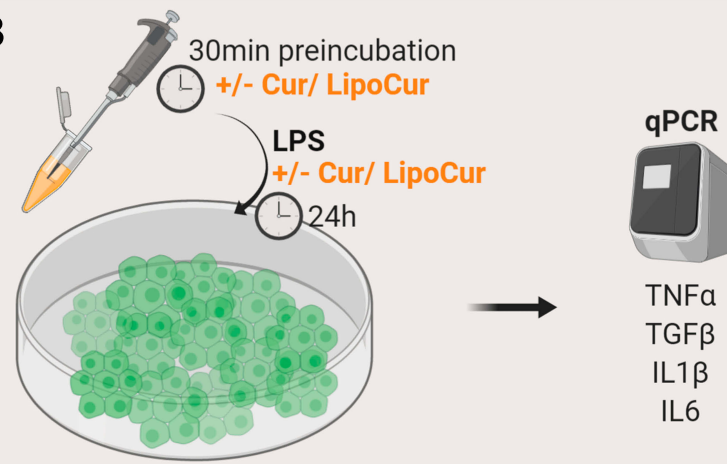

human microglia (HMC3)
C

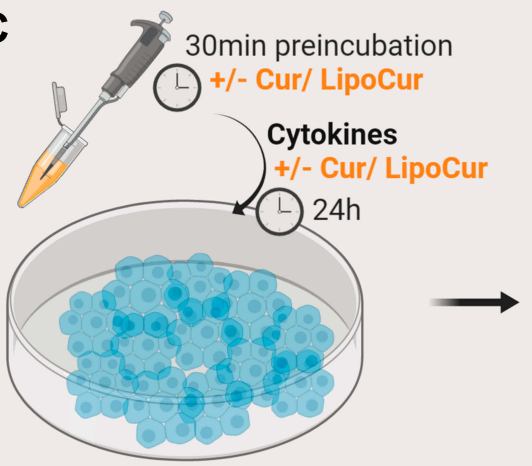

human astrocytes (SVGA)

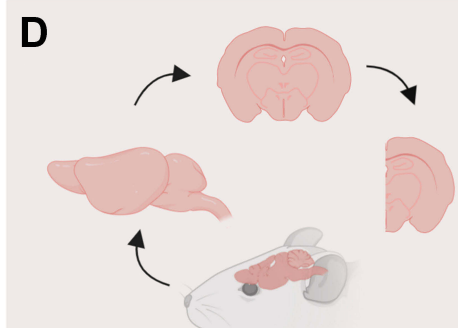

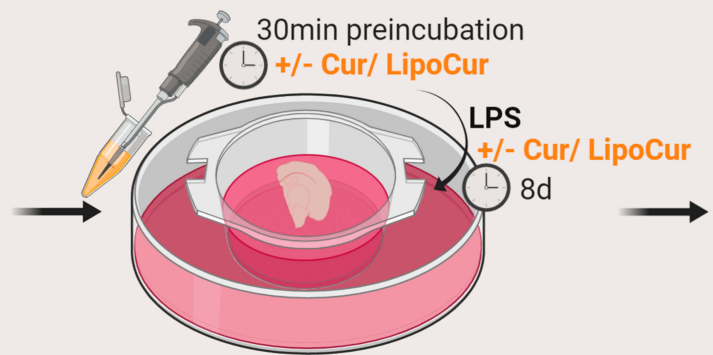

murine organotypic brain slices

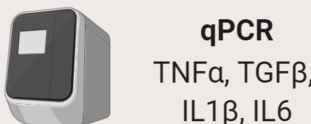

IL1 $\beta$, IL6

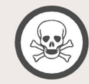

Cytotoxicity

IHC

IHC
Vim, Nes, Fib, GFAP
NF, MBP

Figure I Graphical depiction of brain cell activation and the experimental cell culture-based models. (A) Activated by tissue damage, microglial cells enter their activated state characterized by an amoeboid shape and the production of pro-inflammatory cytokines such as ILI $\beta$, IL6, TNF $\alpha$ and TGF $\beta$. These cytokines exert neuroinflammatory reaction and turn astrocytes to a reactive state in which they upregulate the expression of gliosis specific genes. (B) Human microglial cells HMC3 were stimulated with LPS to induce an inflammatory reaction, which is amongst others represented by the production of inflammatory cytokines like ILI $\beta$, IL6, TNF $\alpha$ and TGF $\beta$ I. Expression of these mediators was analyzed on mRNA level by qPCR to investigate the modulating effects of liposomal-encapsulated curcumin (LipoCur) in comparison to free curcumin and empty liposomes on the inflammatory response of microglial cells. (C) In order to transfer the inflammatory response to astrocytes, human SVGA astrocytes were stimulated with a combination of the microglia produced cytokines ILI $\beta$, TNF $\alpha$ and TGF $\beta$ I (with or without co-stimulation with LipoCur or free curcumin), and genes that are known to contribute to reactive gliosis and glial scar formation (Nestin, Vimentin, Tenascin-C, Fibronectin) were analyzed by qPCR. (D) To study the cellular reactions in their tissue environment, we stimulated murine organotypic brain slices with LPS and analyzed by cytotoxicity assay, qPCR and immunohistochemistry whether LPS effects could be ameliorated by co-incubation with LipoCur or free curcumin.

liposomes were purified by centrifugation to separate free curcumin from the nano-formulation. In brief, a first centrifugation step for $15 \mathrm{~min}$ at $9000 \mathrm{xg}$ at room temperature (Centrifuge 5418, Eppendorf AG, Hamburg, Germany) separated free curcumin agglomerates (pellet) from the liposomal formulation (supernatant), whereas two ultracentrifugation steps for $1 \mathrm{~h}$ at $100,000 \mathrm{xg}$ at $4^{\circ} \mathrm{C}$ (OptimaTM L-90K, Beckman-Coulter, Brea, CA) separated the liposomal formulation (pellet) from the remaining free drug (supernatant). The final pellet was resuspended in $1.2 \mathrm{~mL}$ ultrapure water. The pellet from the first centrifugation as well as the supernatants from the ultracentrifugation steps were kept for indirect quantification of the encapsulated curcumin concentration by highperformance liquid chromatography (HPLC, see below).

For freeze-drying of the liposomes (see paragraph Freeze-drying of liposomal formulations), the ultrapure water for the rehydration of the lipid film as well as the resuspension of the liposomal pellet after centrifugation was supplemented by $10 \%$ trehalose in the final protocol. To evaluate the most suitable condition for freeze-drying, at first different concentrations of trehalose were tested against each other and evaluated by their physicochemical properties. 


\section{Freeze-Drying of Liposomal Formulations}

Freshly produced liposomes were freeze-dried using a vacuum freeze-dryer (Drywinner 8, Heto-Holten A/S, Allerod, Denmark). In brief, the samples were frozen from room temperature to $-45^{\circ}$ (cycle 1) under 1 bar over a period of $3 \mathrm{~h}$ and $30 \mathrm{~min}$. After that, primary drying was performed at $-45^{\circ} \mathrm{C}$ for $15 \mathrm{~min}$ (under $0.8 \mathrm{bar}$ pressure), at $-15^{\circ} \mathrm{C}$ for $3 \mathrm{~h}$ (under $0.1 \mathrm{bar}$ pressure) and then at $-10^{\circ} \mathrm{C}$ for $12 \mathrm{~h}$ (under 0.1 bar pressure). Finally, the secondary drying was carried out at $10^{\circ} \mathrm{C}$ for $5 \mathrm{~h}$ (under 0.1 bar pressure). The entire freeze-drying cycle took $23 \mathrm{~h}$ and $45 \mathrm{~min}$. Freeze-dried samples were kept at $4{ }^{\circ} \mathrm{C}$ under vacuum until further usage.

The mass yield of curcumin loaded liposomes was determined by gravimetric analysis of the dried liposomes dispersions. Briefly, liposome formulation without the addition of trehalose were freeze-dried as described before. The weight of the dried liposomes was measured and the mass yield (\%) was calculated using Equation 1.

$$
\text { mass yield }(\%)=\frac{\text { weight of } 200 \mu \text { L liposomes } x 100}{\text { theoretical weight of } 200 \mu \text { L liposomes }}
$$

\section{Physico-Chemical Properties of Liposomes}

\section{Dynamic Light Scattering (DLS) and Laser-Doppler Electrophoresis}

All samples of liposomes (empty or curcumin loaded) were analyzed three times for the size $(\mathrm{nm})$, polydispersity index and zeta potential $(\mathrm{mV})$ by Dynamic Light Scattering (DLS) (BIC 90 plus, Brookhaven Instruments Corp., Holtsville, NY) followed by Laser-Doppler Anemometry, based on the electrophoretic mobility (Zetasizer Nano ZS, Malvern, UK), right after production. The measurements were performed following a 1:100 dilution of all the samples with ultrapure water, at $25^{\circ} \mathrm{C}$. For long-term stability tests, aliquots of freeze-dried samples, stored at $4^{\circ} \mathrm{C}$ under vacuum, were rehydrated and analyzed over the time for up to 9 months in storage with three technical replicates, $\mathrm{n}=3$.

\section{High-Performance Liquid \\ Chromatography (HPLC)}

The percentage of encapsulation efficiency $(\% \mathrm{EE})$ was determined indirectly (see equation 2) of the curcumin content lost during the centrifugation respective ultracentrifugation steps by a previously validated HPLC method (Agilent LC1100 Series, Agilent Technologies Santa Clara, CA, with OpenLab CDS LC ChemStation version C.01.05 as the software). Moreover, the drug loading capacity was determined by direct quantification of encapsulated curcu$\mathrm{min}$ in the final liposomal formulations, as described in equation 3. The elution was carried out in an isocratic mode with sample volumes of $10 \mu \mathrm{L}$ and a flow rate of $0.8 \mathrm{~mL} / \mathrm{min}$ at ambient temperature and a wavelength of $427 \mathrm{~nm}$. The separation was performed on an Xterra RP8 column $\left(5 \mu \mathrm{m}, 4.6 \times 250 \mathrm{~mm}\right.$, end-capped Waters ${ }^{\circledR}$, Zellik, Belgium) and a mobile phase consisting of $10 \%$ ultrapure water and $90 \%$ phase E1 (41\% acetonitrile, $23 \%$ methanol and $36 \%$ acidified ultrapure water). Curcumin concentrations were calculated on the basis of linear calibration functions and with regard to the dilution factor. Standards, as well as the curcumin pellet (centrifugation), were prepared in absolute ethanol and the supernatants (ultracentrifugation) and the liposomal formulation were diluted 1:3 with ethanol respective 1:10 with ethanol supplemented with $0.5 \%$ Triton-X. All samples were run as duplicates.

$$
\% E E=\frac{\text { (total amount of curcumin })-(\text { free curcumin })}{\text { total amount of curcumin }} * 100 \%
$$

$$
\begin{aligned}
& \text { drug loading capacity }\left(\frac{\mu g \text { curcumin }}{m g \text { liposomes }}\right) \\
& =\frac{\text { amount of curcumin in } 200 \mu \text { Liposomes } *[\mu g]}{\text { weight of } 200 \mu \text { liposomes }[\mathrm{mg}]}
\end{aligned}
$$

\section{Transmission Electron Microscopy}

The morphology of the freeze-dried liposomes, empty as well as curcumin loaded, was analyzed by Transmission Electron Microscopy (TEM) after 9 months of storage as described before. ${ }^{54}$ In brief, $3 \mu \mathrm{L}$ of diluted sample was added on a freshly glow discharged continuous carbon-coated copper grid (Electron Microscopy Sciences, Hatfield, PA) and liposomes were allowed to adhere to the surface for $10 \mathrm{sec}$. The excess sample was blotted off from the side with filter paper, 5 $\mu \mathrm{L}$ of half saturated uranyl acetate solution was added twice and removed two sec after each incubation. After drying at room temperature, grids were transferred to a JEOL JEM 1400Plus TEM operating at $100 \mathrm{kV}$ (Tokyo, Japan). Images were digitally recorded on a $4 \mathrm{kx} 4 \mathrm{k}$ digital camera (F416, TVIPS, Germany).

\section{In vitro Curcumin Release from Liposomes}

The in vitro drug release profiles of liposomes were studied with the dialysis method. In brief, freeze-dried 
curcumin loading liposomes were rehydrated in a final volume of $400 \mu \mathrm{L}$ ultrapure water and vigorously vortexed. To determine the total amount of curcumin available at time $\mathrm{t}=0(\mathrm{X} 1)$, a $10 \mu \mathrm{L}$ sample volume was kept for HPLC quantification. In parallel, $50 \mu \mathrm{L}$ of the sample were taken to quantify free curcumin outside the liposomes. For this, the $50 \mu \mathrm{L}$ were diluted in $950 \mu \mathrm{L}$ ultrapure water and centrifuged $15 \mathrm{~min}$ at $9000 \mathrm{xg}$ at room temperature. The remaining pellet, considered to be free curcumin, was diluted in $1 \mathrm{~mL}$ ethanol and the free curcumin content (X2) was quantified via HPLC. Therefore, the difference of concentration between $\mathrm{X} 1$ and $\mathrm{X} 2$ represents the actual amount of curcumin present inside the liposomes at $\mathrm{t}=0$.

Then, the remaining $340 \mu \mathrm{L}$ of rehydrated samples were taken in dialysis bags (Biotech CE Tubing, MWCO $20 \mathrm{kDA}$, Fisher Scientific) and dialyzed against $5 \mathrm{~mL}$ ultrapure water containing $20 \%$ ethanol and $0.5 \%$ Tween80 (1:50 acceptor/donator volume ratio to obtain sink condition) at $37^{\circ} \mathrm{C}$, stirred at $25 \mathrm{rpm}$ for 7 days. At certain time points $(0 \mathrm{~h}, 1 \mathrm{~h}, 3 \mathrm{~h}, 6 \mathrm{~h}, 8 \mathrm{~h}, 10 \mathrm{~h}, 24,48 \mathrm{~h}, 72 \mathrm{~h}, 96$ $\mathrm{h}$ and $120 \mathrm{~h}$, respectively) $250 \mu \mathrm{L}$ sample volume was taken from the receptor compartment and replaced by the same volume of fresh receptor compartment media. The concentration of curcumin was then determined via HPLC and the accumulated drug release over the time was calculated taking X2 into account (as it will be directly released from the dialysis bag).

\section{Cell Lines and Cell Culture}

The human fetal astrocyte cell line SVGA was kindly provided by the group of Christine Hanssen Rinaldo, University Hospital of North Norway with the permission of W.J. Altwood. ${ }^{55,56}$ The human microglia cell line HMC3 was purchased from the American Type Culture Collection (ATCC, Manassas, Virginia, USA). Cells were cultured in Dulbecco's modified Eagle's medium (DMEM; Life Technologies, Carlsbad, CA, USA) supplemented with 10\% fetal bovine serum (FBS; PAN-Biotech GmbH, Aidenbach, Germany), 1\% Penicillin-Streptomycin (10,000 U/mL; Thermo Fisher Scientific, Waltham, MA, USA) and $2 \mathrm{mM}$ additional L-Glutamine (Thermo Fisher Scientific). Cells were routinely checked for Mycoplasma contamination by nuclei staining and mycoplasma-specific PCR.

\section{Cell Stimulations}

Cells were seeded in 6-well plates (Sarstedt, Nürnbrecht, Germany) 1 day prior to stimulation. Curcumin (Sigma-
Aldrich) was dissolved in ultrapure water at a stock concentration of $100 \mathrm{mM}$ and further diluted to $0.01 \mu \mathrm{M}$ with cell culture medium (DMEM $+10 \%$ FCS, 1\% Pen/Strep and $2 \mathrm{mM}$ glutamine, see above) shortly prior to stimulations. For all stimulations, $0.01 \mu \mathrm{M}$ curcumin or the corresponding amount of liposomal encapsulated curcumin (or empty liposomes) were added to the cells $30 \mathrm{~min}$ prior to further stimulation and maintained for the whole stimulation period. This procedure is termed "preincubation" in the following. The inflammatory reaction of microglia was induced by stimulation with $100 \mathrm{ng} / \mathrm{mL}$ lipopolysaccharide (LPS) of Salmonella typhimurium (Sigma-Aldrich) for $24 \mathrm{~h}$. Astrocytes were stimulated for $24 \mathrm{~h}$ with each $10 \mathrm{ng} / \mathrm{mL}$ recombinant human tumor necrosis factor $\alpha$ (TNF $\alpha)$, transforming growth factor $\beta 1$ (TGF $\beta 1$ ) and interleukin $1 \beta$ (IL1 $\beta$ ) (all Immunotools, Friesoythe, Germany) to induce gliosis reaction. Supernatants were collected for viability assays, cells were counted to determine effects on proliferation, and cells were lysed to isolate RNA (for quantitative reverse transcription PCR, qPCR).

Brain slices were pre-incubated with $0.01 \mu \mathrm{M}$ curcumin or the corresponding amount of liposomal encapsulated curcumin (or empty liposomes) $30 \mathrm{~min}$ prior to further stimulation with $100 \mathrm{ng} / \mathrm{mL}$ LPS to induce inflammatory conditions for 8 days, while media and stimuli were changed on days 1 , 2 and 5, and supernatants collected for subsequent cytotoxicity analysis on days 2 and 8 in vitro (div). After 8 days of stimulation, slices were either lysed for RNA isolation or fixed and embedded for immunohistochemistry.

\section{Liposome Cell Uptake Assay}

Liposomes were stained with $0.25 \%$ nile red (SigmaAldrich) for $24 \mathrm{~h}$ at room temperature. Free nile red was removed from liposomes by centrifugation at $3000 \mathrm{xg}$ for $30 \mathrm{~min}$ in filter membranes (Amicon, Sigma-Aldrich, molecular cutoff: $10,000 \mathrm{MWCO}$ ). $10^{6} \mathrm{HMC} 3$ or SVGA cells were seeded on glass cover slips and grown for $24 \mathrm{~h}$. Stained liposomes (or the nile red staining solution for control) were applied to the cells for $24 \mathrm{~h}$ in concentrations corresponding to $0.01 \mu \mathrm{M}$ free curcumin. Cells were fixed with 4\% paraformaldehyde (PFA, in phosphate-buffered saline, PBS), rinsed with PBS (3x), incubated with Alexa Fluor 647 labelled wheat germ agglutinin (Thermo Fisher scientific, 1:200) for $1 \mathrm{~h}$, rinsed with PBS (3x). Then, nuclei were counterstained with 4',6-diamidino-2-phenylindole (DAPI, Sigma-Aldrich, $30 \mathrm{~min}$ ) and cover slips were embedded after rinsing with PBS and distilled 
water using Shandon Immumount (Thermo Fisher scientific). Cover slips were inspected and documented using an Axiovert 200M microscope with Apotome (Zeiss, Oberkochem, Germany).

\section{Cell Viability}

To measure viability, 7000 HMC3 or SVGA cells/well were seeded on 96-well plates and grown for $24 \mathrm{~h}$. Then, media were changed to DMEM containing 10\% FBS plus respective stimuli or alone as a positive control. After $72 \mathrm{~h}$ incubation, proliferation was determined by the measurement of tetrazolium salt WST-1 cleavage (Roche, Mannheim, Germany) regarding ISO 10993-5 and normalized to unstimulated control ( 2 individual wells for each stimulus, as technical replicates, biological replicates as indicated in the respective figure legends).

\section{Organotypic Brain Slices}

For the generation of organotypic brain slices, female Sox10flox hGFAP:creeRT2 mice (16-24 weeks old; the genetic modification was not relevant for the experimental procedure) were sacrificed by cervical dislocation and brains were carefully removed from the skull and rinsed in artificial cerebrospinal fluid (aCSF, $2 \mathrm{mM} \mathrm{CaCl}_{2}, 10$ $\mathrm{mM}$ D-Glucose, $1.3 \mathrm{mM} \mathrm{MgCl} 2,5 \mathrm{mM} \mathrm{KCl}, 124 \mathrm{mM}$ $\mathrm{NaCl}, 26 \mathrm{mM} \mathrm{NaHCO}$ ). Mouse brains were obtained and used in agreement with the local Ethics Committee "Ministerium für Energiewende, Landwirtschaft, Umwelt und ländliche Räume des Landes Schleswig-Holstein“" [authorization V 242-70056/2015(91-7/15)] and in accordance with the German Tierschutzgesetz (BGBI. I S. 1206, BGBI. I S. 1308) and the European directive 2010/63/EU. Tissue slices of $400 \mu \mathrm{m}$ were obtained using a digital tissue slicer (Stölting, Wood Dale, IL). In brief, brains were dissected to obtain the cerebral cortex regions from both hemispheres, placed on wet filter papers, which were fixed below the blade of the slicer using adhesive film. To ensure minimal surface tension, the blade was wetted with aCSF before every cut. ${ }^{57}$ Slices were prepared, separated and controlled under a binocular microscope and placed on membrane inserts (PICM03050, Millicell ${ }^{\circledR}-\mathrm{CM} 0.4 \mu \mathrm{m}$, Merck Millipore, Burlington, MA, USA) in 6-well plates. Wells contained $1.2 \mathrm{~mL}$ of MEM/HEPES (Thermo Fisher Scientific), supplemented with $24 \%$ horse serum (Sigma Aldrich), 1\% glutamine, $1 \%$ penicillin and streptomycin, 6 $\mathrm{mg} / \mathrm{mL}$ glucose and $168 \mu \mathrm{g} / \mathrm{mL}$ sodium hydrogen phosphate, allowing brain slices to be provided by nutrients from the bottom and $\mathrm{CO}_{2}$ from the top [protocol based and adjusted on previous reports]. ${ }^{57-59}$

\section{Cytotoxicity Assay}

To analyze the protective effects of liposomal and free curcumin on murine brain slices, slices were challenged with $100 \mu \mathrm{g} / \mathrm{mL}$ LPS, in combination or not with $0.01 \mu \mathrm{M}$ free or liposomal curcumin respective empty liposomes. As a positive control, brain slices were treated with 83.2 $\mathrm{ng} / \mathrm{mL}$ Digitonin solution instead of stimulation. Supernatants were collected and cytotoxicity was analyzed using the CytoTox-Fluor ${ }^{\mathrm{TM}}$ Cytotoxicity Assay (Promega, Madison, WI) as described previously ${ }^{60}$ on days 2 and 8 in vitro.

\section{Immunohistochemistry}

After stimulation ( 8 days), brain slices were briefly washed in PBS, fixed in 4\% paraformaldehyde (in Tris-buffered saline, TBS) overnight, equilibrated in $30 \%$ sucrose (in TBS, until slices sink down), embedded and frozen in Shandon Cryomatrix (Thermo Fisher Scientific) and cut in $10 \mu \mathrm{m}$ cryosections. Sections were washed with $0.1 \%$ Tween in TBS (TBS-T), blocked for autofluorescence with 1\% sudan black (Merck, in 70\% ethanol) and for unspecific binding with $0.5 \%$ glycine $/ 0.5 \%$ bovine serum albumin (in TBS-T), incubated with primary antibodies overnight at $4{ }^{\circ} \mathrm{C}$, washed $3 \mathrm{x}$, incubated with Alexa Fluor 488 or 555 labelled secondary antibodies against respective host species (donkey IgG, 1:800, Thermo Fisher scientific) at $37^{\circ} \mathrm{C}$ for $1 \mathrm{~h}$, washed ( $2 \mathrm{x}$, TBS-T, $1 \mathrm{x}$ TBS), nuclei were counterstained with DAPI (Sigma-Aldrich) and slides embedded after washing in TBS-T and water with Shandon Immumount (Thermo Fisher Scientific). For secondary antibody controls, primary antibodies were omitted (compare Figure S1). Commercially available primary antibodies were anti-GFAP (glial fibrillary acidic protein; rabbit, 1:100; DAKO, Glostrup, Denmark, Z0334), antifibronectin (rabbit, 1:400; Novus biologicals, Wiesbaden, Germany; NBP1-91258), anti-MBP (myelin basic protein; mouse, 1:400; Novus, NBP2-22121), anti-nestin (mouse, 4D11, 1:200; Novus, NBP1-92717) and anti-vimentin (chicken, 1:200; Novus, NB300-223). The monoclonal antibody against neurofilament-M (clone $2 \mathrm{H} 3$ ) developed by $\mathrm{T}$. M Jessell and J. Dodd, HHMI/Columbia University was obtained from the Developmental Studies Hybridoma Bank, created by the NICHD of the NIH and maintained at The University of Iowa, Department of Biology, Iowa 
City, IA 52242. Sections were inspected and documented using an Axiovert 200M Fluorescence Microscope.

\section{Quantitative Reverse Transcription-PCR}

HMC3 or SVGA cells were harvested and tissues homogenized using the QIAzol lysis reagent (Qiagen, Hilden, Germany) and total RNA was isolated following the manufacturer's protocol. Genomic DNA was digested by RNase-free Dnase ( $1 \mathrm{U} / \mu \mathrm{L}$, Promega, Madison, $\mathrm{W}, \mathrm{USA})$, and cDNA synthesis was performed using RevertAid ${ }^{\mathrm{TM}}$ H Minus M-MuLV Reverse Transcriptase (200U/ $\mu$ L, Thermo Fisher Scientific, Waltham, MA, USA). TaqMan primer probes and TaqMan ${ }^{\mathrm{TM}}$ Gene expression Master Mix (Thermo Fisher Scientific) were used to analyze samples with the ABI PRISM 7500 Sequence detection system. Analyzed genes were gapdh (human: Hs99999905_m1, mouse: Mm99999915_g1), il6 (human: Hs00985639_m1, mouse: Mm00446190_m1), ill $\beta$ (human: Hs01555410_m1, mouse: Mm00434228_m1), tnfo (human: Hs00174128_m1, mouse: Mm00443258_m1), tgf $\beta$ (human: Hs00171257_m1, mouse: Mm01178820_m1), tenascin (human: Hs01115665_m1), nestin (human: Hs0070120_s1), and fibronectin (human: Hs00277509_m1). Cycle of Threshold values (CT) were measured, and $\Delta \mathrm{CT}$ values $=\mathrm{CT}[$ gene of interest $]-\mathrm{CT}[\mathrm{GAPDH}]$ were calculated . Due to the logarithmic reaction mode, a $\Delta \mathrm{C}_{\mathrm{T}}$ value of 3.33 corresponds to one magnitude lower gene expression compared to GAPDH. For stimuli-induced mRNA regulation
$\Delta \Delta \mathrm{CT}$ values were calculated: $\Delta \Delta \mathrm{CT}=2^{-}(\Delta \mathrm{CT}[$ stimulus $]-$ $\Delta \mathrm{CT}[$ control]).

\section{Statistical Analysis}

All values are shown as mean values \pm standard deviation (SD). Statistical analysis was performed using GraphPad Prism $^{\circledR} 5$ (GraphPad Software, Inc. Version, CA). Asterisks or rhombs indicate statistical significance, whereas $p$ values $<0.05$ were considered as significant. Different statistical analyses were applied in the function of experiments as reported in each figure legend.

\section{Results}

\section{Preparation and Characterization of Liposomes}

For the development of an effective curcumin delivery system for the alleviation of brain implant-induced foreign body reactions in the brain, a liposomal formulation using $80.6 \%$ (w/v) DPPC, $15.6 \%(\mathrm{w} / \mathrm{v})$ cholesterol and 3.8\% (w/v) curcumin was employed. Empty and curcumin loaded liposomes were produced by hydration of the lipid-thin film technique, followed by multiple times extrusion. Particle size analysis of liposomes encapsulating curcumin showed a size, PdI and Zeta Potential (ZP) of $180 \pm 34 \mathrm{~nm}, 0.170 \pm 0.040$ respective $-26.1 \pm 14.0 \mathrm{mV}$ (Table 1) on the day of preparation.

For long-term storage of liposomal formulations, trehalose was added to the final formulation as a cryo-protectant.

Table I Characterization of Empty and Curcumin Loaded Liposomes (LipoCur), Before and After Freeze-Drying

\begin{tabular}{|l|l|l|l|l|l|l|}
\hline & $\begin{array}{l}\text { Size } \\
{[\mathrm{nm}]}\end{array}$ & PdI & $\begin{array}{l}\text { ZP } \\
{[\mathrm{mV}]}\end{array}$ & $\begin{array}{l}\text { DL } \\
{[\%]}\end{array}$ & $\begin{array}{l}\text { MY } \\
{[\%]}\end{array}$ \\
\hline $\begin{array}{l}\text { LipoCur } \\
\text { (wo Tre, before FD) }\end{array}$ & $180 \pm 34$ & $0.17 \pm 0.04$ & $-26.1 \pm 14.0$ & $21.4 \pm 1.8$ & $78 \pm 18$ \\
\hline $\begin{array}{l}\text { LipoCur } \\
(w \text { Tre, before FD) }\end{array}$ & $153 \pm 48$ & $0.11 \pm 0.05$ & $-22.6 \pm 6.2$ & n.d. & $68 \pm 6$ \\
\hline $\begin{array}{l}\text { LipoCur } \\
(w \text { Tre, after FD) }\end{array}$ & $164 \pm 22$ & $0.25 \pm 0.10$ & $-29.3 \pm 3.8$ & n.d. & n.d. & n.d. \\
\hline $\begin{array}{l}\text { Empty liposomes } \\
(w \text { Tre, before FD) }\end{array}$ & $158 \pm 3$ & $0.12 \pm 0.02$ & $-17.7 \pm 0.2$ & n.d. & n.d. \\
\hline $\begin{array}{l}\text { Empty liposomes } \\
(w \text { Tre, after FD) }\end{array}$ & $219 \pm 82$ & $0.30 \pm 0.10$ & $-17.2 \pm 3.8$ & n.d. & n.d. \\
\hline
\end{tabular}

Notes: Curcumin loaded liposomes (LipoCur) were analyzed prior to freeze-drying (FD) with or without substitution of $10 \%$ trehalose (Tre), and with trehalose substitution after freeze-drying regarding size (hydrodynamic diameter), polydispersity index (Pdl), zeta potential (ZP), for comparison, data of empty liposomes (with Trehalose) are shown below before freeze-drying and after freeze-drying. Drug loading (DL), mass yield (MY) and encapsulation efficacy (EE) were measured in freshly prepared liposomes, DL and MY only in freshly prepared, drug-loaded liposomes without trehalose (n.d.: not determined). Mean values of $n=3$ measurements from one batch \pm SD are shown. 
To evaluate the most suitable trehalose concentration that does not alter the size, ZP or PdI after freeze-drying, different trehalose concentrations have been added to the liposomal formulations and physicochemical properties have been evaluated before (Figure 2A) and after freeze-drying (Figure 2B). With the final concentration of $10 \%$ trehalose, sizes of $153 \pm$ $48 \mathrm{~nm}$ and $164 \pm 22 \mathrm{~nm}$, PdI of $0.11 \pm 0.05$ and $0.25 \pm 0.10$, as well as $\mathrm{ZP}$ of $-22.6 \pm 6.2 \mathrm{mV}$ and $-29.3 \pm 3.8 \mathrm{mV}$ have been measured of curcumin loaded liposomes before respective after the freeze-drying process. Empty liposomes showed comparable size $(158 \pm 3 \mathrm{~nm})$ and $\mathrm{PdI}(0.12 \pm 0.02$ $\mathrm{mV})$, and less negative $\mathrm{ZP}(17.7 \pm 0.2)$ than curcumin loaded liposomes before freeze-drying (with trehalose added) and a slightly bigger size $(219 \pm 82 \mathrm{~nm})$ and $\mathrm{PdI}(0.30 \pm 0.10)$ as well as less negative $\mathrm{ZP}(-17.2 \pm 3.8 \mathrm{mV})$ after freeze-drying (Table 1).

With this final formulation, the curcumin encapsulation efficiency (EE) and drug loading capacity (DL) were evaluated by HPLC quantification on the day of preparation. As seen in Table 1, EE values and DL of $68 \pm 6 \%$ respective $21.4 \pm 1.8 \%$ were obtained for liposomes without trehalose, and an EE $46 \pm 9 \%$ for liposomes containing trehalose. The total mass yield that was determined by HPLC and lyophilization was $78 \pm 18 \%$ (Table 1).

Regarding the long-term stability of fabricated liposomes, freeze-dried samples were stored in a vacuum at $4^{\circ} \mathrm{C}$ for up to 180 days and analyzed for their size, PdI and $\mathrm{ZP}$ and compared to the values directly after the freezedrying process (Figure 2C-E). Sizes slightly increased over the time from $180 \mathrm{~nm}$ to $240 \mathrm{~nm}$ (directly vs 180 days after freeze-drying), whereas only after 120 days significant changes to the fresh freeze-dried formulation could be noted. However, the ZP (Figure 2D) and the size distribution (Figure 2E) of the liposomal formulations did not vary over the time. In summary, 180 days old liposomes did not significantly differ from freshly produced formulations in their size, PdI and ZP. Thereafter, using the dialysis method, the in vitro drug release of curcumin was performed and evaluated by HPLC. The individual drug release profile of $n=3$ technical replicates is depicted in Figure 2F. After $10 \mathrm{~h}$, liposomes released $26.9 \% \pm 4.5$ of their curcumin cargo and a maximum with $34.8 \% \pm 5.9$ was released after $24 \mathrm{~h}$, which was followed by a sustained release drug profile over the time.

Concomitant with the above-described physicochemical characterization, we analyzed the morphology of the freezedried liposomes (LipoCur as well as empty LP) by Transmission Electron Microscopy (TEM) after 180 days of storage, and similar sizes could be evaluated visually (Figure $3 \mathrm{~A}$ and $\mathrm{B}$ ) as previously measured by DLS (Figure 2C). All vesicle types tended do have a spherical shape, with similar size distributions. Some of them seemed to be dented or hollow, putatively due to the preparation process with uranyl acetate for TEM.

Taken together, we could successfully establish a protocol for the liposomal encapsulation of curcumin, yielding favorable physicochemical properties, satisfying encapsulation efficiencies and long-term stability of the produced liposomes.

\section{Cellular Uptake and Effects of Liposomal Curcumin on Viability of Human Astrocytes and Microglia Cells}

To study the uptake of liposomes by cells, empty liposomes (LP) and curcumin loaded liposomes (LipoCur) were fluorescently labeled with nile red and incubated with human microglia cells (HMC3) and astrocytes (SVGA) for $24 \mathrm{~h}$. The final liposome concentration of $0.18 \mu \mathrm{g} / \mathrm{mL}$ corresponded to a final concentration of 0.01 $\mu \mathrm{M}$ curcumin. We observed that both cell types efficiently incorporated labeled liposomes (Figure 3C). Using infrared labelled wheat germ agglutinin which binds to and marks cell membranes, we could clearly demonstrate that red-labelled liposomes were located within the cell boundaries in the cytoplasm. However, as nile red labels specifically the liposomal structures, but not the curcumin itself, we could not compare the uptake of liposomes to uptake or effects of free curcumin.

Next, we investigated to what extend empty and curcumin loaded liposomes influenced the viability of cultivated brain cells. Therefore, we stimulated microglia and astrocytes with empty LP, LipoCur and, for comparison, free curcumin (free Cur) for 3 days with concentrations ranging from $0.0001 \mu \mathrm{M}$ to $20 \mu \mathrm{M}$ curcumin (and corresponding amounts of loaded or empty liposomes). As shown in Figure 3D, concentrations up to $1 \mu \mathrm{M}$ did not significantly impair cell viability in both cell types. Empty and curcumin loaded liposomes showed widely comparable effects in this well-tolerated concentration range. Empty LP did significantly decrease cell viability from $90 \mu \mathrm{g} / \mathrm{mL}$ on (corresponding to a curcumin load of $5 \mu \mathrm{M}$ for LipoCur), while LipoCur was slightly less toxic in these high concentrations. However, for further experiments, we used amounts corresponding to $0.01 \mu \mathrm{M}$ curcumin, being more than 2 orders of magnitude below this 
A
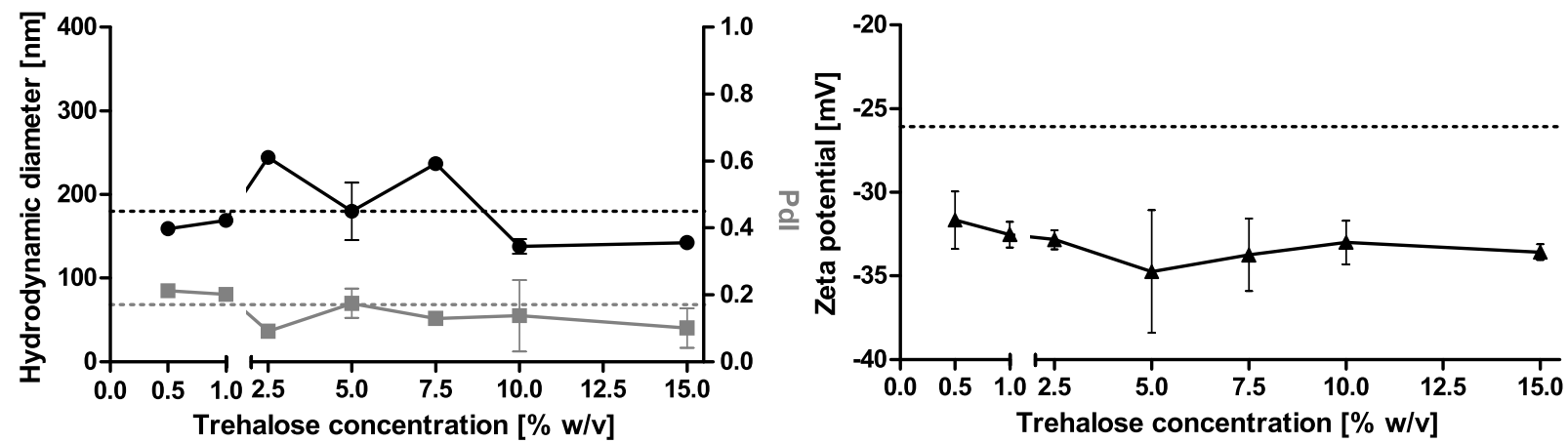

B
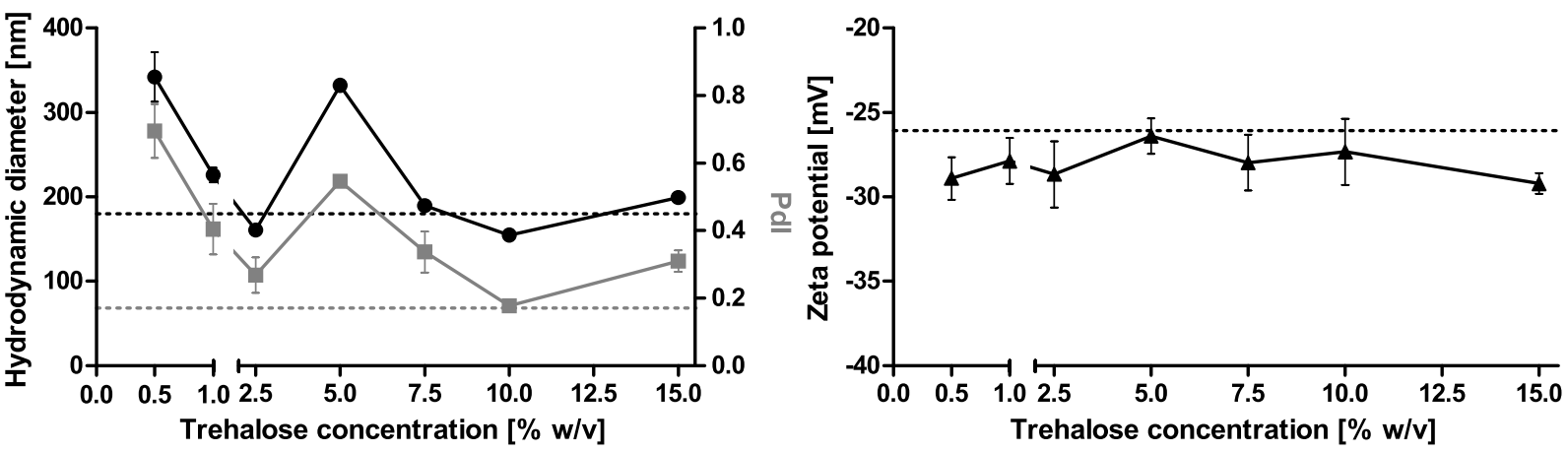

C

D
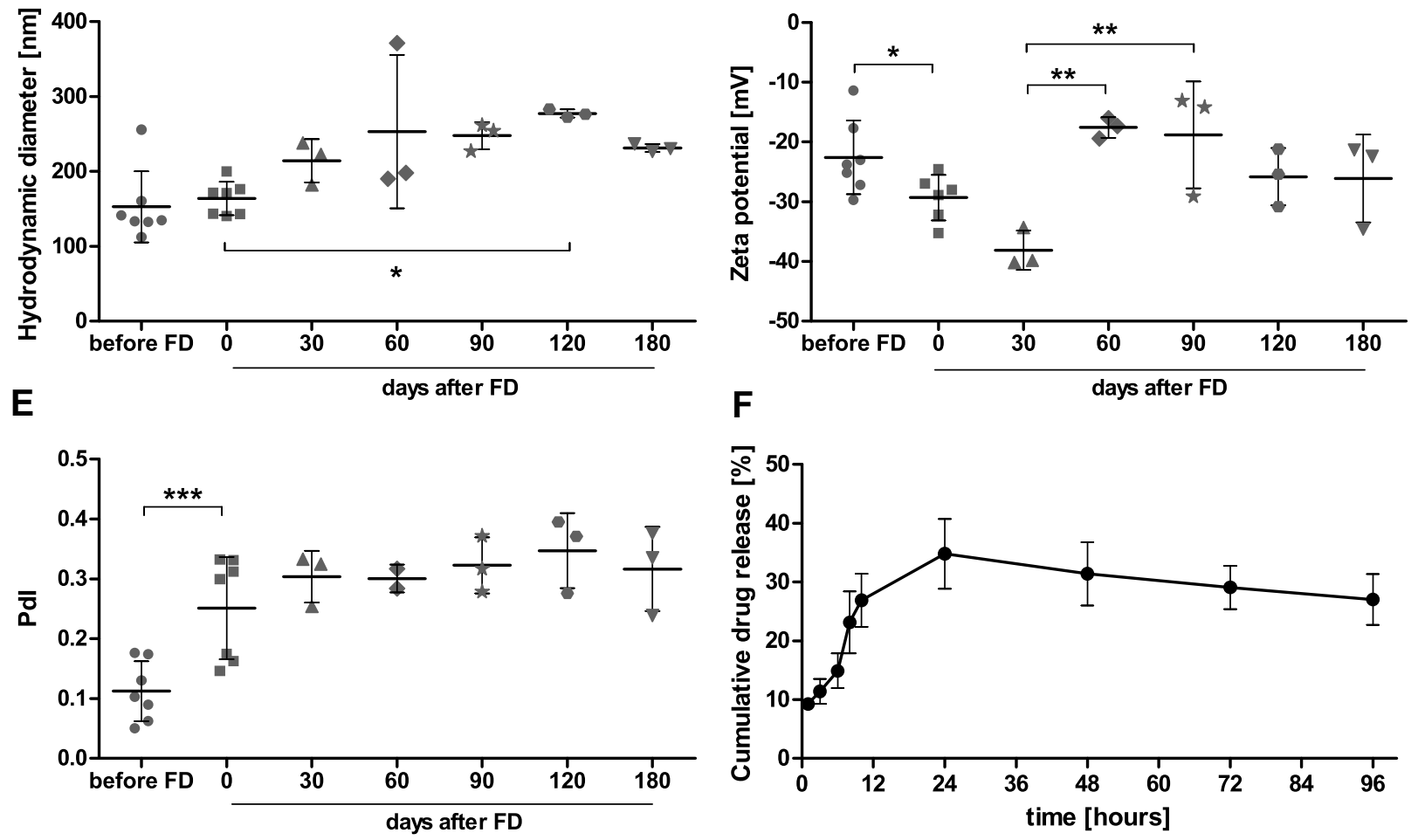

Figure 2 Physicochemical properties of curcumin loaded liposomes. Hydrodynamic diameter, polydispersity index (PdI) and zeta potential were determined of curcumin loaded liposomes substituted with different concentrations of trehalose before $(\mathbf{A})$ and after (B) freeze-drying $(\mathrm{n}=3$ batches). Long-term analysis of hydrodynamic diameter (C), zeta potential (D) and PdI (E) of the freeze-dried liposomes using 10\% trehalose revealed stability for at least 180 days. ( $\mathrm{n}=3$ batches) (F) Curcumin loaded liposomes showed efficient sustained drug release over $96 \mathrm{~h}$. The instability of curcumin is mirrored by decreasing cumulative curcumin concentrations after prolonged time periods $(n=3)$. The statistical significance of the long-term stability was determined by One-Way-ANOVA followed by Bonferroni's multiple comparison test, comparing all days after freeze-drying with one another. A two-tailed paired $t$-test was employed to test for significance between liposomes before and directly after freeze-drying. $*_{p}<0.05, * * p<0.005$, $* * * p<0.001$. 


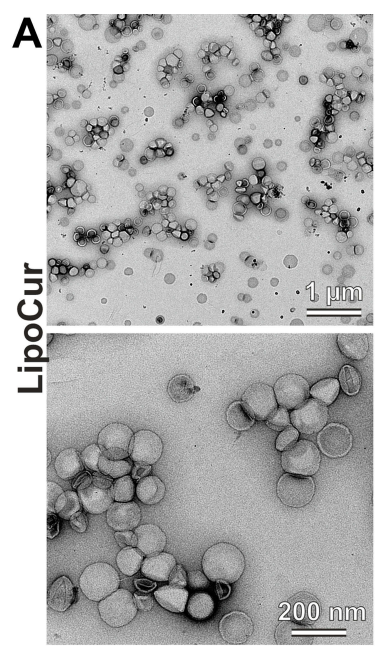

C
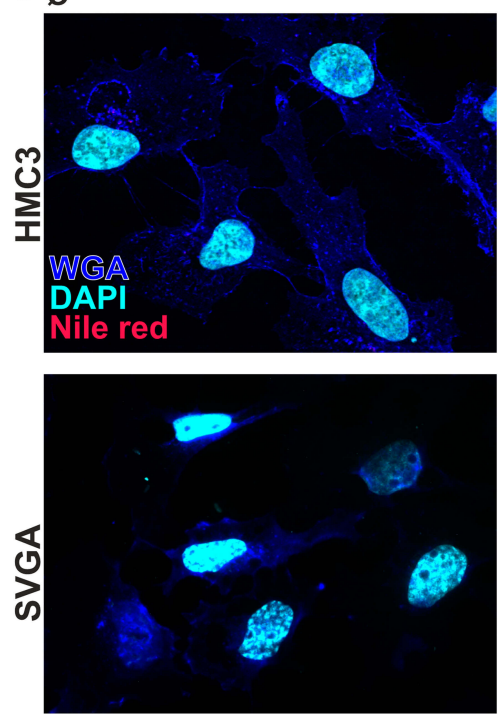

D $\mathrm{HMC3}$

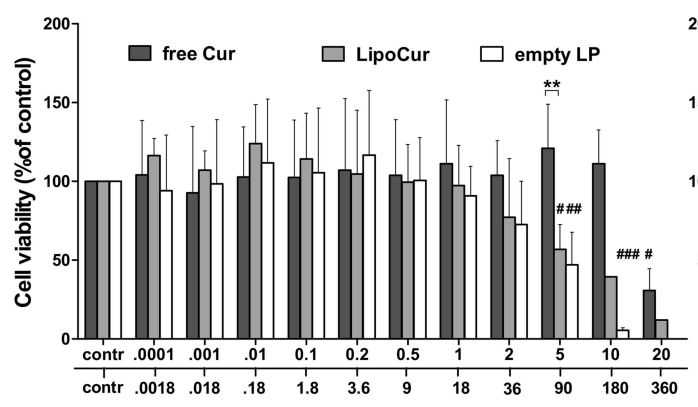

B
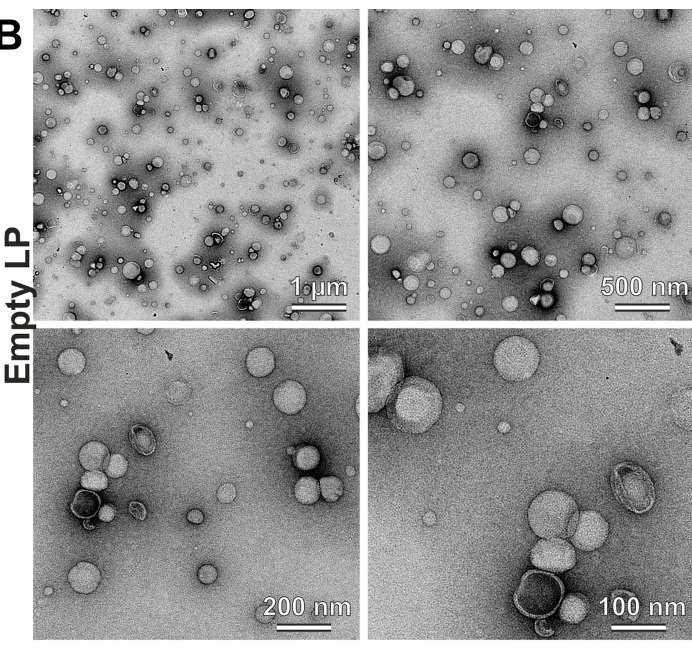

LipoCur

Empty LP
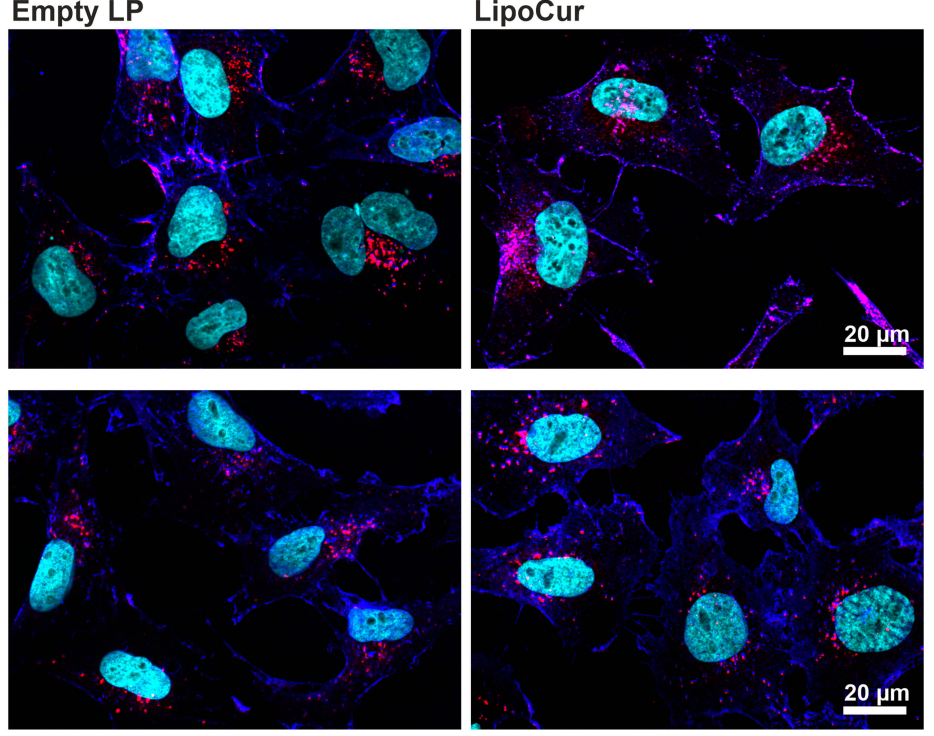

SVGA

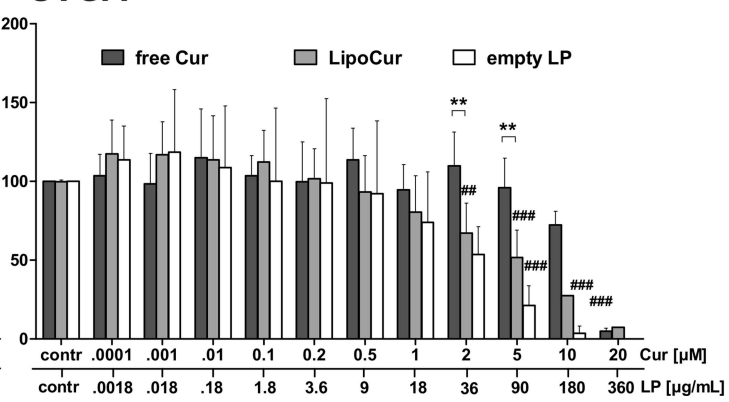

Figure 3 Ultrastructure of curcumin loaded and empty liposomes, cellular uptake and cell viability of HMC3 microglia cells and SVGA astrocytes upon incubation with liposomal and free curcumin. (A) Curcumin loaded liposomes and (B) empty liposomes were visualized by transmission electron microscopy (TEM) at different magnifications. Similar average sizes and size distributions could be obtained as with DLS measurements after 180 days. (C) Liposomes were labelled red using nile red, and cellular uptake was monitored after $24 \mathrm{~h}$ incubation with human microglia cells HMC3 or human astrocytes SVGA. Nuclei were counterstained with DAPI (shown in cyan) and membrane structures with Alexa Fluor-647 labelled wheat germ agglutinin (shown in blue) to visualize the localization of the red labelled liposomes within the cell boundaries. Representative images from $n=2$ independent experiments, scale bars indicate $20 \mu \mathrm{m}$. (D) To test the influence of liposomal curcumin on cell viability, human microglia cells (HMC3, left) or astrocytes (SVGA, right) were incubated with different concentrations of free curcumin (free Cur), curcumin loaded liposomes (LipoCur) containing the same amounts of curcumin (a final concentration of $I \mu M$ curcumin corresponds to $I 8$ $\mu \mathrm{g} / \mathrm{mL}$ loaded liposomes) or the corresponding amounts of empty liposomes (empty LP) for 3 days. Viability was determined in duplicates using a WST-assay for $\mathrm{n}=4-10$ independent experiments, graphs show mean values \pm SD. For HMC3 microglia, there was no significant reduction in viability to be observed up to $10 \mu \mathrm{M}$ for free curcumin (IC50 I9.6 $\mu \mathrm{M}$ ), and up to I $\mu \mathrm{M}$ for liposomal curcumin (IC50 $5.3 \mu \mathrm{M}$ ). Empty liposomes only reduced microglia viability starting from $90 \mu g / \mathrm{mL}$ (IC50 $86.4 \mu g / \mathrm{mL}$ ). SVGA cells showed quite similar results while being a bit more sensitive, with IC50 values of II.6 $\mu \mathrm{M}$ for free curcumin, $2.1 \mu \mathrm{M}$ for liposomal curcumin and $36.4 \mu g / \mathrm{mL}$ for empty liposomes. Statistical significant differences to unstimulated controls (corresponding to $100 \%$ ) were analyzed by One-Way-ANOVA followed by Dunnett's multiple comparison test and are indicated by rhombs $\left({ }^{\#} \mathrm{p}<0.05\right.$, ${ }^{\prime \prime} \mathrm{p}<0.0 \mathrm{I}$,

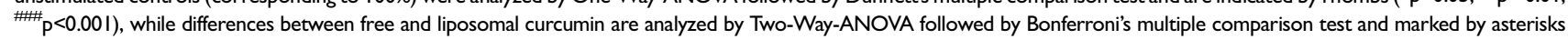
$(* * \mathrm{p}<0.01)$. 
toxic range. Free curcumin was well tolerated at high concentrations $(5-10 \mu \mathrm{M})$ but was also toxic at $20 \mu \mathrm{M}$ for both cell lines.

Taken together, empty and curcumin loaded liposomes were efficiently taken up by microglia and astrocytes and did not impair cell viability at concentrations up to $1 \mu \mathrm{M}$.

\section{Effects of Liposomal Curcumin on Neuroinflammation and Reactive Astrogliosis in vitro}

Our concept of liposomal encapsulation of curcumin aimed to provide a drug formulation for local brain administration to attenuate foreign body reactions towards brain implants. Therefore, we tested in a next step if liposomal curcumin can alleviate neuroinflammatory and reactive astrogliosis processes in vitro. As an experimental model for neuroinflammation (Figure 1B), we stimulated microglia cells with lipopolysaccharide from $S$. typhimurium (100 ng/mL), with or without pre-incubation with LipoCur and, for comparison, free curcumin or empty LP for $24 \mathrm{~h}$. LPS stimulation yielded a typical change to amoeboid morphology (Figure 4A) and induced expression of the cytokines IL6, IL1 $\beta$, TGF $\beta$ and TNF $\alpha$ (Figure 4B, black bars). These effects could be clearly reduced by pre-incubation with LipoCur, and also by free curcumin (Cur). In addition, although not being significantly different in direct comparison, LipoCur yielded frequently higher levels of significance in reducing LPS effects than free Cur. However, due to high variations in the extent of microglia activation (compare error bar of LPS stimulation and LPS + empty LP), effects were not always significant, but stimulations with LPS and LipoCur frequently yielded less induction of inflammatory cytokines than LPS stimulations alone or LPS together with empty LP. In comparison, mRNA expression of analyzed cytokines was much more robust in controls that were not challenged with LPS, and neither free nor liposomal curcumin nor empty liposomes had any effect on expression levels.

As an experimental model for astrocyte reactivity, we used a cytokine cocktail (IL1 $\beta$, TGF $\beta$ and TNF $\alpha, 10 \mathrm{ng} /$ $\mathrm{mL}$ each, $24 \mathrm{~h}$ ) to stimulate human astrocytes (Figure 1C). This cocktail affected astrocyte morphology, yielding, for example, nuclear deformation and alteration in cell size and shape. Again, this effect was almost completely abolished by pre-incubation with LipoCur or free Cur (Figure 4C). As a choice of gliosis-associated genes, we analyzed mRNA expression of tenascin C, fibronectin and nestin, and observed that cytokine-mediated induction of these genes could be significantly reduced by preincubation with LipoCur or free curcumin, reaching or reaching almost levels of controls without cytokine cocktail (Figure 4D). Again, LipoCur reached higher significance levels in gene expression reduction in comparison to free curcumin, thus attenuating more robust the reactive gliosis reaction.

Taken together, our liposomal curcumin formulation was as effective as or even more potent than free curcumin in reducing microglia and astrocyte reactivity.

\section{Effects of Liposomal Curcumin on Viability and LPS Response of Organotypic Murine Brain Slices}

As cellular reactions are strongly influenced by their local tissue environment and cell neighbors, we decided to investigate in the next step the beneficial effects of LipoCur in comparison to free curcumin and empty LP in LPS-challenged organotypic brain slices (Figure 1D). Using $400 \mu \mathrm{m}$ slices from one murine brain cortex region, the reactions of a whole-brain tissue with all its cells types towards various stimuli could be analyzed.

First, we could show that LipoCur and free Cur could restore LPS-reduced viability of organotypic brain slices to control levels at early ( $2 \mathrm{div})$ and late $(8 \mathrm{div})$ time points of incubations (Figure 5A). In the next step, we analyzed the expression of inflammatory markers IL1 $\beta$, IL6, TNF $\alpha$ and TGF $\beta$ on mRNA levels at 8 div. IL1 $\beta$, IL6 and TNF $\alpha$ were induced upon LPS stimulation, and these effects were in trend or even significantly mildened by pre-incubation with LipoCur, and to lesser extend free Cur (Figure 5B). When interpreting these results, one has to keep in mind, that the microglial cells represent only one small portion of cells within the slices, so that smaller effects and higher variations should be expected. The cytokine TGF $\beta$ was not induced by LPS stimulation at 8 div, so that pre-incubation with LipoCur or free Cur did not yield any effects.

Finally, we used fluorescence immunohistochemistry to visualize LPS-mediated cellular changes and restoration by pre-incubation with LipoCur or free Cur (Figure 6). Stainings of neurofilament (NF) revealed that the neuronal network was clearly affected by LPS stimulation at 8 div, and these effects could be almost completely diminished by pre-incubation with LipoCur (or free Cur), but not by empty LP. Furthermore, markers for reactive astrogliosis 

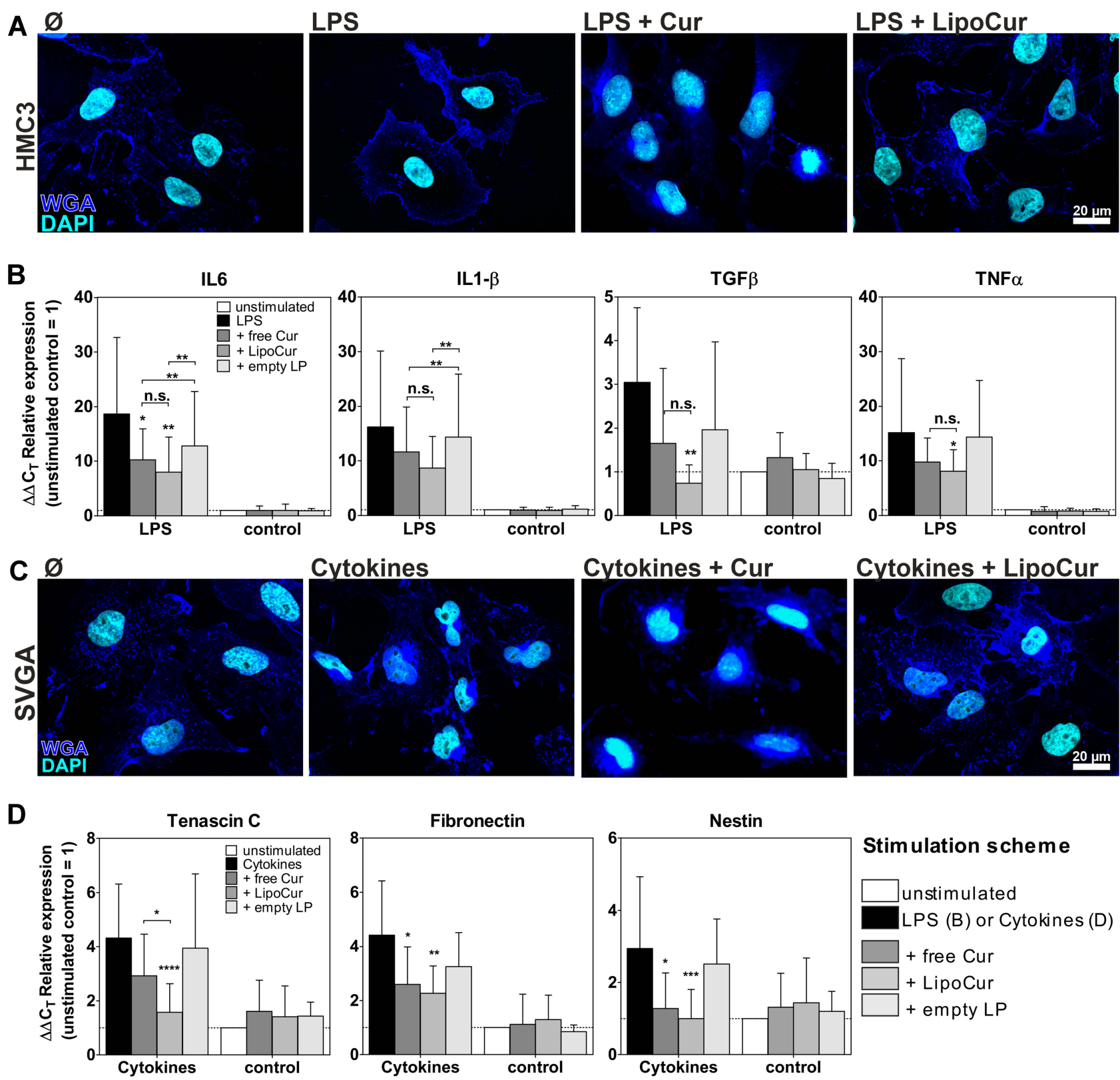

\section{Stimulation scheme}

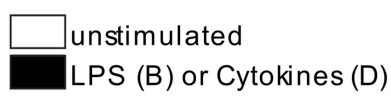

Figure 4 Effects of liposomal and free curcumin on experimental models of neuroinflammation (A, B) and reactive gliosis (C, D). (A) Upon stimulation with I00 ng/mL LPS for $24 \mathrm{~h}, \mathrm{HMC} 3$ cells showed the typical amoeboid morphology of reactive microglia, which could be alleviated by pre-incubation with $0.0 \mathrm{I} \mu \mathrm{M}$ free (Cur) or liposomal curcumin (LipoCur). Representative images of $\mathrm{n}=2$ independent stimulations, scale bar indicate $20 \mu \mathrm{m}$. (B) Stimulation with $100 \mathrm{ng} / \mathrm{mL} \mathrm{LPS}$ for $24 \mathrm{~h}$ also induced expression of proinflammatory cytokines IL6, ILI $\beta$, TNF $\alpha$ and TGF $\beta$, as monitored by qPCR. These effects could be reduced by pre-incubation with $0.0 \mathrm{I} \mu \mathrm{M}$ curcumin and, in trend even more effective, by corresponding amounts of LipoCur. Please note that due to high variations of LPS-induced cytokine expression curcumin and LipoCur mediated reduction is not always significant, but trends can be seen for all investigated cytokines. Graphs show $\Delta \Delta C T$ values representing the $n$-fold expression in comparison to unstimulated controls. In non-inflammatory conditions (without LPS stimulation), neither curcumin, nor LipoCur, nor empty liposomes induced any changes in expression of these genes. (C) Stimulation with a combination of ILI $\beta$, TNF $\alpha$ and TGF $\beta(10 \mathrm{ng} / \mathrm{mL}$ each $)$ for $24 \mathrm{~h}$ served to induce activation of human astrocyte cell line SVGA. Cells show nuclear deformations as a sign of cellular stress. Effects were almost completely abolished upon pre-incubation with $0.01 \mu M$ free curcumin or LipoCur. Representative images from $n=2$ experiments, scale bar indicates $20 \mu \mathrm{m}$. (D) As representatives for reactive astrogliosis associated genes, expression of nestin, tenascin $\mathrm{C}$ and fibronectin was analyzed by qPCR and found to be induced upon stimulation with ILI $\beta$, TNF $\alpha$ and TGF $\beta(I 0 \mathrm{ng} / \mathrm{mL}$ each) for $24 \mathrm{~h}$. These effects were significantly alleviated by pre-incubation with $0.0 \mathrm{I} \mu \mathrm{M}$ curcumin or LipoCur. LipoCur showed higher significance levels compared to soluble curcumin and was in case of tenascin $C$ even significantly more effective. In "normal" conditions (without cytokine stimulation), neither curcumin, nor LipoCur, nor empty liposomes induced any changes in expression of these genes. Shown are mean values \pm SD from $n=5-15$ independent experiments, asterisks indicate significant changes of stimulations compared to LPS control (B) and cytokines (D) or between the groups under the brackets. Data have been analyzed by Two-Way-ANOVA followed by Bonferroni's multiple comparison test $\left({ }^{*} p<0.05, * * p<0.001,{ }^{* * *} p<0.001,{ }^{*} * * * p<0.0001\right)$.

(GFAP, nestin, fibronectin, vimentin) showed strong immunoreactivity in LPS-stimulated slices, and again these effects were alleviated by LipoCur and free Cur, but not empty LP. However, stainings for oligodendrocytes (MBP, myelin basic protein), hardly showed any differences between LPS stimulations and controls, and within 


\section{A 2 div}

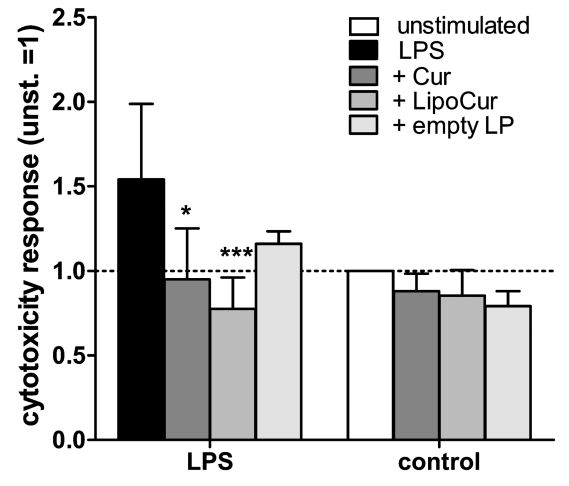

B

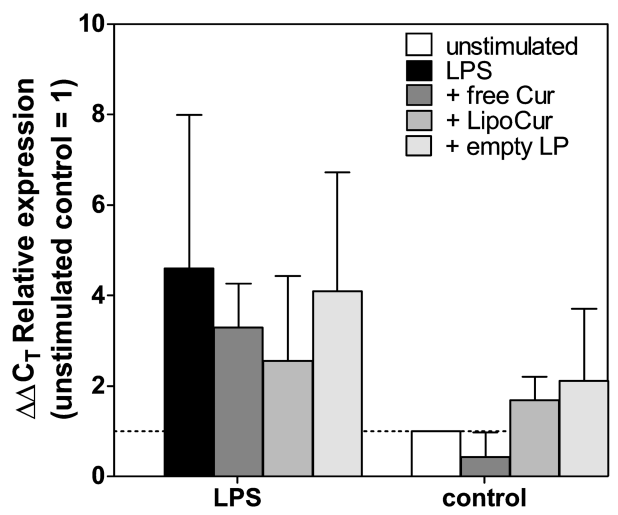

TGF $\beta$

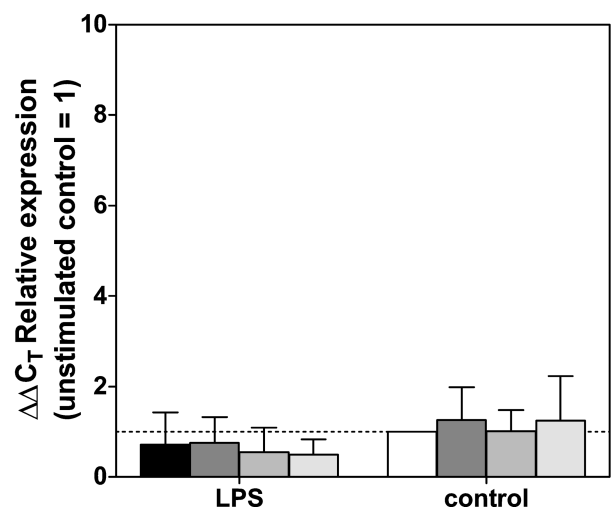

8 div

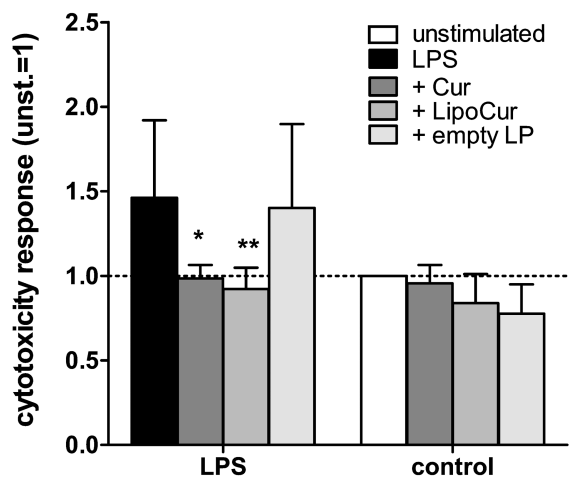

IL1 $\beta$

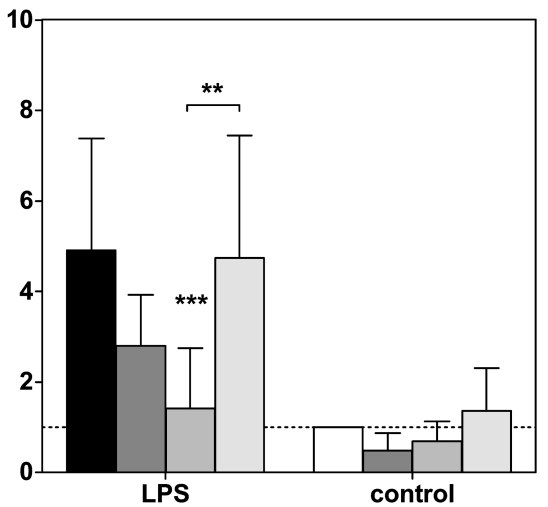

TNF $\alpha$

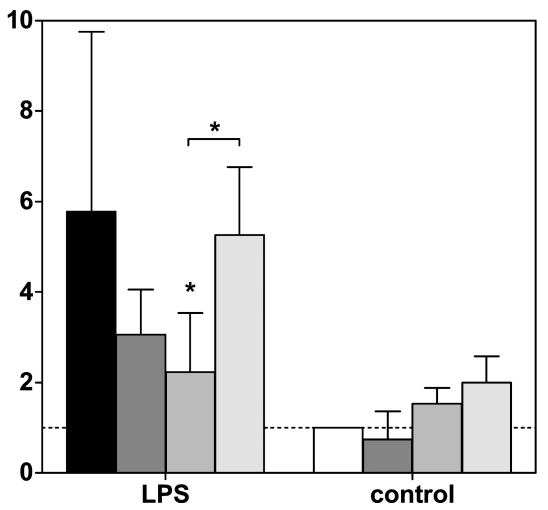

Figure 5 Effects of liposomal and free curcumin on LPS-challenged organotypic brain slices, regarding cytotoxicity (A) and alteration of mRNA expression of proinflammatory cytokines (B). (A) Murine acute brain slices were treated with $100 \mu \mathrm{g} / \mathrm{mL}$ LPS with or without pre-incubation with $0.01 \mu M$ free curcumin or LipoCur for 8 days, and cytotoxicity was measured from supernatants after 2 and 8 div by Cytotoxicity assay. LPS stimulation induced cytotoxicity (black bar) was completely abolished (compare unstimulated control: white bar) by simultaneous stimulation with LipoCur or free Cur, but not by empty liposomes (empty LP, grey bars). Neither curcumin nor LipoCur nor empty LP had significant effects on cytotoxicity in single stimulations (without LPS, grey bars). Measured extinctions were normalized to unstimulated control $(=I)$, and mean values of 3 respective 5 independent experiments $( \pm S D)$ are shown for cytotoxic effects after2 div respective 8 div. Asterisks indicate significant changes to LPS-stimulated slices $\left({ }^{*} p<0.05, * * p<0.001, * * * p<0.001\right.$ ), analyzed by Two-Way-ANOVA followed by Bonferroni's multiple comparison test. (B) Analyses of effects of LipoCur and free Cur on the expression of cytokines revealed that both could at least in trend reduce LPS-induced upregulation of inflammatory genes ILI $\beta$, IL6, and TNF $\alpha$. TGF $\beta$ was not induced by LPS at $8 \mathrm{div}$, and consequently not affected by LipoCur or free Cur. Graphs show mean $\triangle \triangle C T$ values \pm SD representing the n-fold expression in comparison to unstimulated controls. Data have been analyzed for statistical significance by Two-Way-ANOVA followed by Bonferroni's multiple comparison test. Asterisks indicate significant changes to LPS-stimulated slices or between stimulations $\left({ }^{*} p<0.05,{ }^{*} p<0.001, * * *<<0.001\right)$.

there were no effects observed upon stimulation with LipoCur or free Cur. Secondary antibody controls are shown in Figure S1.
Taken together, we could establish a protocol for the liposomal encapsulation of curcumin, yielding liposomes with favorable physicochemical properties, efficient drug 


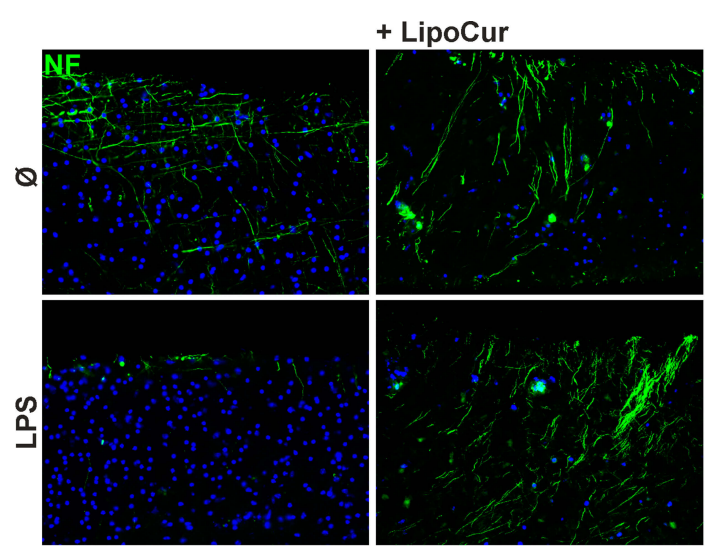

+ empty LP

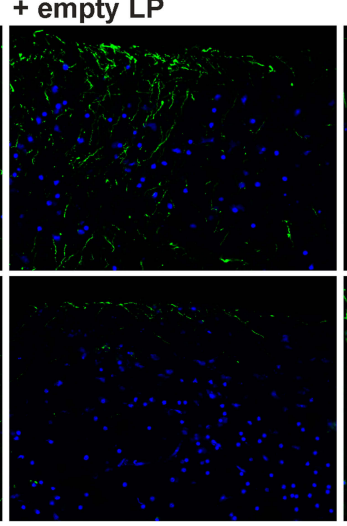

+ free Cur
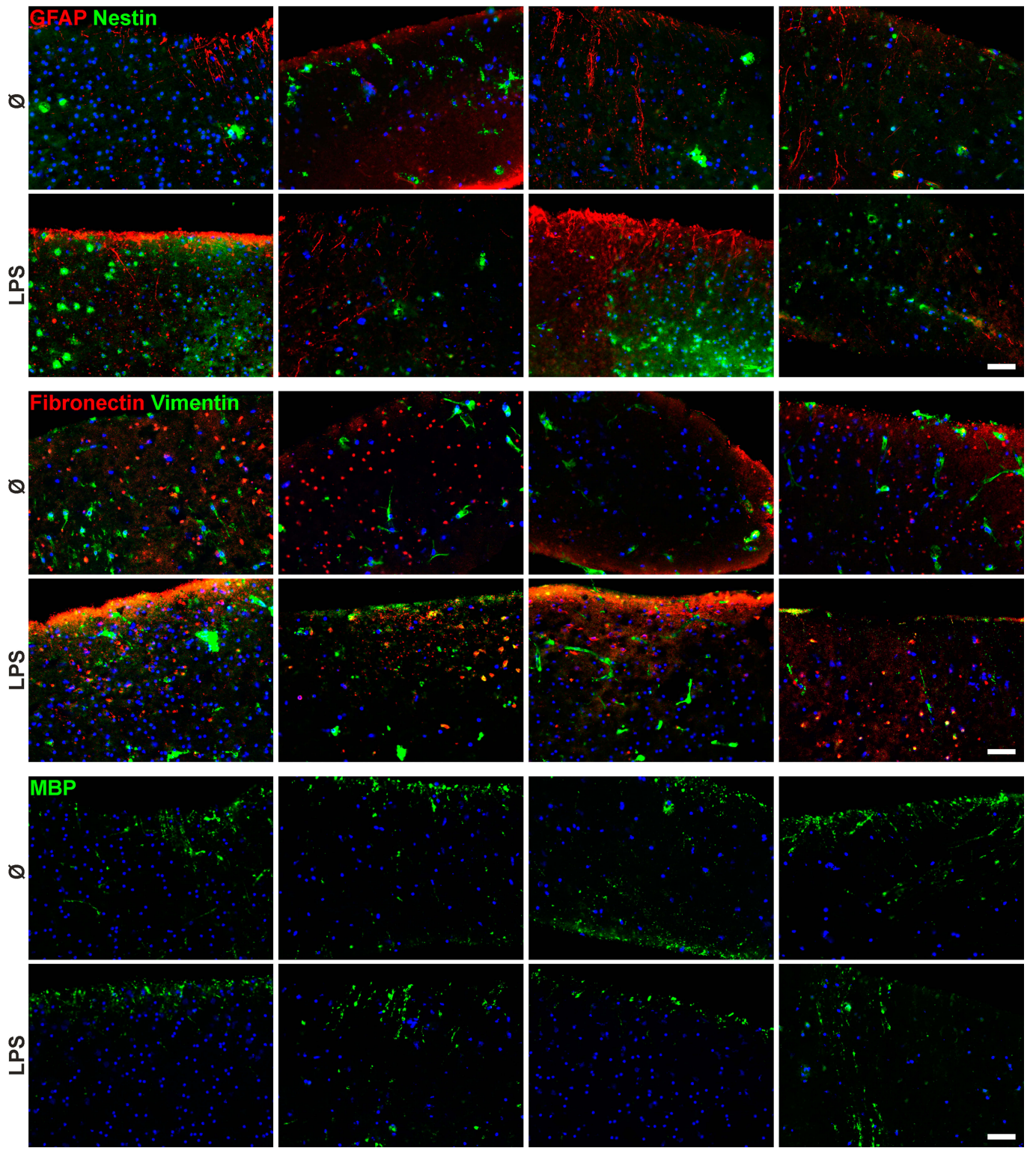

Figure 6 Effects of liposomal (LipoCur) and free Curcumin on LPS-challenged organotypic brain slice morphology. Murine acute brain slices were treated with $100 \mu \mathrm{g} / \mathrm{mL}$ LPS with or without $0.01 \mu \mathrm{M}$ free curcumin or LipoCur for 8 days, slices were cut in $10 \mu \mathrm{m}$ cryosections and immunostained with antibodies against neurofilament-M (NF, neurons), GFAP and nestin (astrocytes, glial precursors, reactive astrocytes), fibronectin and vimentin (reactive astrocytes, glial scarring), as well as MBP (oligodendrocytes). These exemplary pictures of $n=4$ individual experiments show that LipoCur and free curcumin (but not empty liposomes) can effectively restore LPS-mediated loss of neurofilament and attenuate induction of reactive gliosis associated markers like GFAP, nestin, fibronectin and vimentin. Oligodendrocytes marked by MBP staining, however, were hardly affected by LPS stimulation and co-stimulation with LipoCur or free curcumin. Scale Bars indicate $50 \mu \mathrm{m}$, please refer to Figure $\mathrm{SI}$ for secondary antibody controls. 
encapsulation, in vitro drug release and long-term stability. These liposomes (we termed them LipoCur) were taken up into the cytoplasmic cell compartment and were well tolerated by human microglia and astrocytes as well as murine organotypic brain slices. In experimental models for neuroinflammation and reactive astrogliosis, LipoCur could efficiently reduce microglia and astrocyte reactivity, in some settings even more effective than free curcumin.

\section{Discussion}

The natural compound curcumin is a traditional coloring and flavoring food ingredient, and has attracted broad scientific interest due to its possible anti-oxidative and antiinflammatory properties. Aiming to develop a biocompatible, longterm stable nano-formulation of a drug, that may alleviate foreign body reactions provoked by brain implant materials, we gave a chance to curcumin as several previous reports had shown that curcumin treatment reduces the extent of the experimental brain and spinal cord injuries. In animal models of spinal cord injuries, curcumin provided neuroprotection and attenuated microglial and astrocyte reactivity as shown by reduced GFAP-expression levels and Nestin+/GFAP+ glial scar regions as well as reduced expression of proinflammatory cytokines like IL1 $\beta$, IL6, TNF $\alpha$, TGF $\beta 1$ and 2, involving different intracellular signaling pathways. Additionally, curcumin reduced reactive gliosis in the rat brain stem after ethidium bromide injection yielding smaller GFAP + glial scar regions. ${ }^{26-29,61,62}$

However, like a multitude of other drugs, curcumin is quite instable and poorly bioavailable so that the systemic application of the native drug will hardly achieve therapeutically relevant local concentrations. ${ }^{63}$ In first attempts to apply curcumin locally to the brain, poly (vinyl alcohol) (PVA) based implants were loaded with curcumin and implanted into the rodent brain. ${ }^{64}$ In comparison to neat implants, curcumin improved neuronal survival and bloodbrain integrity 4 weeks after implantation, but after 12 weeks, curcumin benefits were lost. ${ }^{64}$ Due to its known beneficial effects in CNS injuries, we chose curcumin as a model drug to develop a formulation that may attenuate glial scarring processes. To provide a long-term stable nano-formulation for curcumin that can be used in implants for local brain therapy (eg entrapped in slowly biodegradable coatings) we encapsulated curcumin into liposomes, as these nano-carriers are widely used and can be easily adapted to specific requirements. ${ }^{42,45,65}$ As an example, the physicochemical properties of liposomes can be tailored by changing the phospholipids themselves or the ratio between them. In their previous work, Roy et al have analyzed the impact of different biomimetic lipids and lipid-combinations such as egg phosphatidylcholine (EPC), dipalmitoylphosphatidylcholine (DPPC) or dipalmitoylphosphatidylglycerol (DPPG) on the stability of liposomes containing curcumin. ${ }^{66}$ Amongst them, DPPC was the most $\mathrm{pH}$-stable one with a sustained drug release at physiological $\mathrm{pH}$. Pre-experiments in our laboratory comparing DPPC with EPC (data not shown) revealed that higher encapsulation efficiencies of curcumin, as well as smaller PdIs, could be obtained using DPPC as the main phospholipid. Consequently, we chose DPPC as the backbone of our liposomal formulation.

To provide adequate storage conditions, the right cryoprotectant must be chosen for the individual phospholipid combination upon freeze-drying. ${ }^{67}$ Based on the work of van Winden and Crommelin as well as Mohammed and colleagues we chose to work with trehalose as cryoprotectant, to ensure stable curcumin nanoformulations with a sufficient EE\% as well as drug release kinetic. ${ }^{68,69}$ With the final supplementation of $10 \%$ trehalose, we could produce a stable liposomal formulation that can be stored up to 6 months at least. Moreover, the sustained release profile of our liposomal formulation at physiological temperatures and $\mathrm{pH}$ with a maximum of only $35 \%$ released curcumin cargo after $24 \mathrm{~h}$ is well aligned with the findings of Roy et al, who highlighted the connection between the lipid transition temperature of DPPC and the slower release kinetics of curcumin liposomes. ${ }^{66}$ Using other lipids than DPPC, such as EPC or SPC for example, Chen et al reported releases of $45-50 \%$ of their total curcumin cargo already after $24 \mathrm{~h}^{46}$ Also, Zhao et al claim that their liposomal formulation of curcumin using soy lecithin released $\approx 50 \%$ after $24 \mathrm{~h}$ and already $80 \%$ of their cargo after $78 \mathrm{~h}^{70}$ Concluding these aspects, we could successfully establish a protocol for liposomal curcumin formulation, using $80.6 \%(\mathrm{w} / \mathrm{w})$ DPPC and $15.6 \%(\mathrm{w} / \mathrm{w})$ cholesterol and $10 \%$ trehalose as a cryoprotectant, and observed beneficial physicochemical properties, efficient drug loading and long-term stability of the liposomes.

To investigate if our liposomal encapsulated curcumin is efficient in preventing glial scar formation, we chose human cell lines of the two major cell types that contribute to these processes, microglia and astrocytes. Therefore, we experimentally split the reactive processes into two steps by activating microglial cells with LPS to analyze pro-inflammatory cytokine expression, and activating astrocytes with a pro- 
inflammatory cytokine mixture typically expressed in glial scarring to analyze glial scarring associated genes. ${ }^{71}$ In vitro, curcumin has previously been shown to reduce the expression of pro-inflammatory mediators and cytokines in activated microglial cells via different signaling pathways. $^{23,25,72-76}$ Curcumin stimulation could also reduce reactivity/activation in astrocytes or astrocytic cell culture models upon different challenges. ${ }^{77-79}$ In accordance with these findings, we could show that our liposomal curcumin formulation could inhibit LPS-induced cytokine expression by human microglial cells, often even more robust than free curcumin. Furthermore, we simulated reactive astrogliosis by stimulating human astrocytes with a cytokine cocktail, and liposomal curcumin could efficiently reduce the cytokinemediated upregulation of gliosis-associated genes, exemplarily ECM components tenascin $\mathrm{C}$ and fibronectin, and the intracellular structure protein nestin.

Very recently, different other approaches have already shown that nanoformulations may improve curcumin stability and applicability, for example, in cancer therapy and brain disease models. ${ }^{34,48,80,81}$ In a spinal cord injury model, Lipodisq-formulated curcumin was shown to be effective in reducing the glial scar formation, and in a demyelination model, curcumin nanoparticles reduced GFAP expression, signs of inflammation and demyelination even a bit more effective than free curcumin. ${ }^{82,83}$ We could also show that our liposomal formulation of curcumin was more effective in inhibiting most of the analyzed proinflammatory and astrogliosis reactions, although differences between free and liposomal curcumin in some aspects were also quite faint. These differences between free and liposomal curcumin might be caused by better bioavailability or higher stability of liposomal curcumin in aqueous solutions, but as we did not test these aspects, we can only speculate on that. However, the efficacy of curcumin nanoformulations needs to be carefully evaluated as sometimes simple monolayer (2D) cell culture models are more susceptible than more complex 3D in vitro models. ${ }^{80}$ Therefore, we used murine brain acute slices from the cerebral cortex as a second test system. We induced reactivity by application of LPS and observed the induction of pro-inflammatory cytokines and reactive gliosis associated genes. In comparable brain slice models, curcumin has previously been shown to protect from $\mathrm{A} \beta 1-42$-induced toxicity, to prevent cell death as well as bilirubin-induced cell damage, and to reduce seizure-like events. ${ }^{84-88}$ In our brain slice model, we could show that our liposomal curcumin formulation could alleviate cytotoxic effects of LPS stimulations, induction of pro- inflammatory cytokines and genes associated with reactive astrogliosis and restore cellular loss, being at least as effective as free curcumin. Thus, we could show in two different test systems (even from different species) that liposomal encapsulated curcumin (LipoCur) can effectively prevent reactive processes that occur in glial scar formation.

\section{Conclusion}

Our liposomal formulation of curcumin can effectively reduce glial scarring associated microglia and astrocyte reactions in human cell culture models as well as murine acute brain slices. Liposomal curcumin is suitable for long-term storage $(\leq 6$ months), well tolerated by healthy cells in relevant concentrations and at least as effective as free (soluble) curcumin in alleviating glial scarring reactions in human astrocytes and microglia as well as in murine acute brain slices.

\section{Abbreviations}

BBB, blood-brain barrier; CT, cycle of threshold; DBS, deep brain stimulation; div, days in vitro; DL, Drug Loading; DLS, Dynamic Light Scattering; DMSO, dimethylsulfoxide; DPPC, dipalmitoylphosphatidylcholine; EE, Encapsulation Efficiency; GAPDH, glyceraldehyde 3-phosphate dehydrogenase; GFAP, glial fibrillary acidic protein; HPLC, High-Performance Liquid Chromatography; IHC, immunohistochemistry; IL1 $\beta$, interleukin $1 \beta$; IL6, interleukin 6; LipoCur, liposomal encapsulated curcumin; LP, liposomes; LPS, lipopolysaccharide; qPCR, quantitative reverse transcription-polymerase chain reaction; MBP, myelin basic protein; MY, mass yield; NF, neurofilament; PdI, Polydispersity Index; SD, standard deviation; TEM, Transmission Electron Microscopy; TGF $\beta 1$, transforming growth factor $\beta 1$; TNF $\alpha$, tumor necrosis factor $\alpha$; Z, zeta potential.

\section{Ethics Approval and Informed Consent}

Mouse brains were obtained and used in agreement with the local Ethics Committee "Ministerium für Energiewende, Landwirtschaft, Umwelt und ländliche Räume des Landes Schleswig-Holstein“" [authorization V 242-70056/2015 (917/15)] and in accordance with the German Tierschutzgesetz (BGBI. I S. 1206, BGBI. I S. 1308) and the European directive 2010/63/EU.

\section{Acknowledgments}

We thank Judith Becker, Martina Burmester, Sonja Dahle, Bettina Facompré, Frank Lichte, Gaby Steinkamp, Laurence 
Collard and Françoise Leonard for expert technical assistance. This work was supported by the Deutsche Forschungsgemeinschaft (DFG, RTG2154, project 7 and 8). PA and the microscopy unit of the Institute of Anatomy, University Kiel, were supported by the DFG SFB877 (project number 125440785; A13 and Z3). The funding bodies did not have any influence on the design of the study.

\section{Author Contributions}

All authors made substantial contributions to conception and design, acquisition of data, or analysis and interpretation of data; took part in drafting the article or revising it critically for important intellectual content; gave final approval of the version to be published; and agree to be accountable for all aspects of the work.

\section{Disclosure}

The authors report no conflicts of interest in this work.

\section{References}

1. Linnaeus C. Species Plantarum. Stockholm: Lars Salvius; 1753.

2. Adiwidjaja J, McLachlan AJ, Boddy AV. Curcumin as a clinically-promising anti-cancer agent: pharmacokinetics and drug interactions. Expert Opin Drug Metab Toxicol. 2017;13(9):953-972. doi:10.1080/17425255.2017.1360279

3. Deguchi A. Curcumin targets in inflammation and cancer. Endocr Metab Immune Disord Drug Targets. 2015;15(2):88-96. doi:10.2174/ 1871530315666150316120458

4. Naksuriya O, Okonogi S, Schiffelers RM, Hennink WE. Curcumin nanoformulations: a review of pharmaceutical properties and preclinical studies and clinical data related to cancer treatment. Biomaterials. 2014;35(10):3365-3383. doi:10.1016/j.biomaterials.2013.12.090

5. Wong KE, Ngai SC, Chan KG, Lee LH, Goh BH, Chuah LH. Curcumin nanoformulations for colorectal cancer: a review. Front Pharmacol. 2019;10:152. doi:10.3389/fphar.2019.00152

6. Gupta SC, Patchva S, Aggarwal BB. Therapeutic roles of curcumin: lessons learned from clinical trials. AAPS J. 2013;15(1):195-218. doi:10.1208/s12248-012-9432-8

7. Yang M, Akbar U, Mohan C. Curcumin in autoimmune and rheumatic diseases. Nutrients. 2019;11(5):E1004. doi:10.3390/nu11051004

8. Farkhondeh T, Samarghandian S, Pourbagher-Shahri AM, Sedaghat M. The impact of curcumin and its modified formulations on Alzheimer's disease. J Cell Physiol. 2019;234(10):16953-16965. doi: $10.1002 /$ jcp. 28411

9. Lim GP, Chu T, Yang F, Beech W, Frautschy SA, Cole GM. The curry spice curcumin reduces oxidative damage and amyloid pathology in an Alzheimer transgenic mouse. J Neurosci. 2001;21 (21):8370-8377. doi:10.1523/JNEUROSCI.21-21-08370.2001

10. Ramkumar M, Rajasankar S, Gobi VV, et al. Demethoxycurcumin, a natural derivative of curcumin abrogates rotenone-induced dopamine depletion and motor deficits by its antioxidative and anti-inflammatory properties in parkinsonian rats. Pharmacogn Mag. 2018;14(53):9-16. doi:10.4103/pm.pm_113_17

11. Tripanichkul W, Jaroensuppaperch EO. Ameliorating effects of curcumin on 6-OHDA-induced dopaminergic denervation, glial response, and SOD1 reduction in the striatum of hemiparkinsonian mice. Eur Rev Med Pharmacol Sci. 2013;17(10):1360-1368.
12. Arora V, Kuhad A, Tiwari V, Chopra K. Curcumin ameliorates reserpine-induced pain-depression dyad: behavioural, biochemical, neurochemical and molecular evidences. Psychoneuroendocrinology. 2011;36(10):1570-1581. doi:10.1016/j.psyneuen.2011.04.012

13. Sun J, Chen F, Braun C, et al. Role of curcumin in the management of pathological pain. Phytomedicine. 2018;48:129-140. doi:10.1016/ j.phymed.2018.04.045

14. Drion CM, Borm LE, Kooijman L, et al. Effects of rapamycin and curcumin treatment on the development of epilepsy after electrically induced status epilepticus in rats. Epilepsia. 2016;57(5):688-697. doi:10.1111/epi.13345

15. Bavarsad K, Barreto GE, Hadjzadeh MA, Sahebkar A. Protective effects of curcumin against ischemia-reperfusion injury in the nervous system. Mol Neurobiol. 2018.

16. Zhang Y, Fang M, Sun Y, et al. Curcumin attenuates cerebral ischemia injury in Sprague-Dawley rats and PC12 cells by suppressing overactivated autophagy. J Photochem Photobiol B. 2018;184:1-6. doi:10.1016/j.jphotobiol.2018.05.010

17. Deng Y, Lu X, Wang L, et al. Curcumin inhibits the AKT/NF-kappaB signaling via $\mathrm{CpG}$ demethylation of the promoter and restoration of NEP in the N2a cell line. AAPS J. 2014;16(4):649-657. doi:10.1208/ s12248-014-9605-8

18. Dong W, Yang B, Wang L, et al. Curcumin plays neuroprotective roles against traumatic brain injury partly via $\mathrm{Nrf2}$ signaling. Toxicol Appl Pharmacol. 2018;346:28-36. doi:10.1016/j.taap.2018.03.020

19. Kim HY, Park EJ, Joe EH, Jou I. Curcumin suppresses Janus kinase-STAT inflammatory signaling through activation of $\mathrm{Src}$ homology 2 domain-containing tyrosine phosphatase 2 in brain microglia. $J$ Immunol. 2003;171(11):6072-6079. doi:10.4049/ jimmunol.171.11.6072

20. Shi X, Zheng Z, Li J, et al. Curcumin inhibits Abeta-induced microglial inflammatory responses in vitro: involvement of ERK1/2 and p38 signaling pathways. Neurosci Lett. 2015;594:105-110. doi:10.1016/j.neulet.2015.03.045

21. Tu XK, Yang WZ, Chen JP, et al. Curcumin inhibits TLR2/4-NFkappaB signaling pathway and attenuates brain damage in permanent focal cerebral ischemia in rats. Inflammation. 2014;37(5):1544-1551. doi:10.1007/s10753-014-9881-6

22. Mhillaj E, Tarozzi A, Pruccoli L, Cuomo V, Trabace L, Mancuso C. Curcumin and heme oxygenase: neuroprotection and beyond. Int J Mol Sci. 2019;20:10. doi:10.3390/ijms20102419

23. Yang Z, Zhao T, Zou Y, Zhang JH, Feng H. Curcumin inhibits microglia inflammation and confers neuroprotection in intracerebral hemorrhage. Immunol Lett. 2014;160(1):89-95. doi:10.1016/j. imlet.2014.03.005

24. Ghasemi F, Bagheri H, Barreto GE, Read MI, Sahebkar A. Effects of curcumin on microglial cells. Neurotox Res. 2019;36(1):12-26. doi:10.1007/s12640-019-00030-0

25. Parada E, Buendia I, Navarro E, Avendano C, Egea J, Lopez MG. Microglial HO-1 induction by curcumin provides antioxidant, antineuroinflammatory, and glioprotective effects. Mol Nutr Food Res. 2015;59(9):1690-1700. doi:10.1002/mnfr.201500279

26. Bondan E, Cardoso C, Martins MF. Curcumin decreases astrocytic reaction after gliotoxic injury in the rat brainstem. Arq Neuropsiquiatr. 2017;75(8):546-552. doi:10.1590/0004-282x20170092

27. Wang YF, Zu JN, Li J, Chen C, Xi CY, Yan JL. Curcumin promotes the spinal cord repair via inhibition of glial scar formation and inflammation. Neurosci Lett. 2014;560:51-56. doi:10.1016/j. neulet.2013.11.050

28. Yuan J, Liu W, Zhu H, et al. Curcumin inhibits glial scar formation by suppressing astrocyte-induced inflammation and fibrosis in vitro and in vivo. Brain Res. 2017;1655:90-103. doi:10.1016/j.brainres.2016.11.002

29. Yuan J, Zou M, Xiang X, et al. Curcumin improves neural function after spinal cord injury by the joint inhibition of the intracellular and extracellular components of glial scar. J Surg Res. 2015;195 (1):235-245. doi:10.1016/j.jss.2014.12.055 
30. Machova Urdzikova L, Karova K, Ruzicka J, et al. The anti-inflammatory compound curcumin enhances locomotor and sensory recovery after spinal cord injury in rats by immunomodulation. Int J Mol Sci. 2015;17:1. doi:10.3390/ijms17010049

31. Lestari ML, Indrayanto G. Curcumin. Profiles Drug Subst Excip Relat Methodol. 2014;39:113-204.

32. Yang KY, Lin LC, Tseng TY, Wang SC, Tsai TH. Oral bioavailability of curcumin in rat and the herbal analysis from Curcuma longa by LC-MS/MS. J Chromatogr B Analyt Technol Biomed Life Sci. 2007;853(1-2):183-189. doi:10.1016/j.jchromb.2007.03.010

33. Liu W, Zhai Y, Heng X, et al. Oral bioavailability of curcumin: problems and advancements. J Drug Target. 2016;24(8):694-702. doi:10.3109/1061186X.2016.1157883

34. Del Prado-Audelo ML, Caballero-Floran IH, Meza-Toledo JA, et al. Formulations of curcumin nanoparticles for brain diseases. Biomolecules. 2019;9:2. doi:10.3390/biom9020056

35. Fereydouni N, Darroudi M, Movaffagh J, et al. Curcumin nanofibers for the purpose of wound healing. J Cell Physiol. 2019;234 (5):5537-5554. doi:10.1002/jcp. 27362

36. Jamwal R. Bioavailable curcumin formulations: A review of pharmacokinetic studies in healthy volunteers. J Integr Med. 2018;16 (6):367-374. doi:10.1016/j.joim.2018.07.001

37. Khan I, Saeed K, Khan I. Nanoparticles: properties, applications and toxicities. Arab J Chem. 2019;12(7):908-931. doi:10.1016/j. arabjc.2017.05.011

38. Hoshyar N, Gray S, Han H, Bao G. The effect of nanoparticle size on in vivo pharmacokinetics and cellular interaction. Nanomedicine. 2016;11(6):673-692. doi:10.2217/nnm.16.5

39. Choi YH, Han HK. Correction to: nanomedicines: current status and future perspectives in aspect of drug delivery and pharmacokinetics. J Pharm Invest. 2019;49(1):201. doi:10.1007/s40005-018-00412-0

40. Bulbake U, Doppalapudi S, Kommineni N, Khan W. Liposomal formulations in clinical use: an updated review. Pharmaceutics. 2017;9:2. doi:10.3390/pharmaceutics9020012

41. Johnston MJ, Semple SC, Klimuk SK, Ansell S, Maurer N, Cullis PR. Characterization of the drug retention and pharmacokinetic properties of liposomal nanoparticles containing dihydrosphingomyelin. Biochim Biophys Acta. 2007;1768(5):1121-1127. doi:10.1016/j.bbamem.2007. 01.019

42. Akbarzadeh A, Rezaei-Sadabady R, Davaran S, et al. Liposome: classification, preparation, and applications. Nanoscale Res Lett. 2013;8(1):102. doi:10.1186/1556-276X-8-102

43. Sabin J, Prieto G, Ruso JM, Hidalgo-Alvarez R, Sarmiento F. Size and stability of liposomes: a possible role of hydration and osmotic forces. Eur Phys J E Soft Matter. 2006;20(4):401-408. doi:10.1140/ epje/i2006-10029-9

44. Al-Jamal WT, Kostarelos K. Liposome-nanoparticle hybrids for multimodal diagnostic and therapeutic applications. Nanomedicine. 2007;2(1):85-98. doi:10.2217/17435889.2.1.85

45. Basnet P, Hussain H, Tho I, Skalko-Basnet N. Liposomal delivery system enhances anti-inflammatory properties of curcumin. J Pharm Sci. 2012;101(2):598-609. doi:10.1002/jps.22785

46. Chen Y, Wu Q, Zhang Z, Yuan L, Liu X, Zhou L. Preparation of curcumin-loaded liposomes and evaluation of their skin permeation and pharmacodynamics. Molecules. 2012;17(5):5972-5987. doi:10. 3390/molecules 17055972

47. Cheng C, Peng S, Li Z, Zou L, Liu W, Liu C. Improved bioavailability of curcumin in liposomes prepared using a $\mathrm{pH}$-driven, organic solvent-free, easily scalable process. RSC Adv. 2017;7 (42):25978-25986. doi:10.1039/C7RA02861J

48. Feng T, Wei Y, Lee RJ, Zhao L. Liposomal curcumin and its application in cancer. Int J Nanomedicine. 2017;12:6027-6044. doi:10.2147/ IJN.S132434

49. Roy A, Saha S, Choudhury A, Bahadur S. Bioenhancement of curcumin by combined approaches of adjuvants and liposomal fabrication. Asian J Pharm. 2016;10:4.
50. Mercanti G, Ragazzi E, Toffano G, Giusti P, Zusso M. Phosphatidylserine and curcumin act synergistically to down-regulate release of interleukin-1beta from lipopolysaccharide-stimulated cortical primary microglial cells. CNS Neurol Disord Drug Targets. 2014;13 (5):792-800. doi:10.2174/1871527313666140414121723

51. Wang Y, Luo J, Li SY. Nano-curcumin simultaneously protects the blood-brain barrier and reduces M1 microglial activation during cerebral ischemia-reperfusion injury. 2 .

52. Marin C, Fernandez E. Biocompatibility of intracortical microelectrodes: current status and future prospects. Front Neuroeng. 2010;3:8. doi:10.3389/fneng.2010.00008

53. Polikov VS, Tresco PA, Reichert WM. Response of brain tissue to chronically implanted neural electrodes. J Neurosci Methods. 2005;148(1):1-18. doi:10.1016/j.jneumeth.2005.08.015

54. Arnold P, Himmels $P$, Weiss S, et al. Antigenic and 3D structural characterization of soluble X4 and hybrid X4-R5 HIV-1 Env trimers. Retrovirology. 2014;11(1):42. doi:10.1186/1742-4690-11-42

55. Henriksen S, Tylden GD, Dumoulin A, Sharma BN, Hirsch HH, Rinaldo $\mathrm{CH}$. The human fetal glial cell line SVG p12 contains infectious BK polyomavirus. $J$ Virol. 2014;88(13):7556-7568. doi:10.1128/JVI.00696-14

56. Schweighardt B, Shieh JT, Atwood WJ. CD4/CXCR4-independent infection of human astrocytes by a T-tropic strain of HIV-1. $J$ Neurovirol. 2001;7(2):155-162. doi:10.1080/13550280152058816

57. Schommer J, Schrag M, Nackenoff A, Marwarha G, Ghribi O. Method for organotypic tissue culture in the aged animal. MethodsX. 2017;4:166-171. doi:10.1016/j.mex.2017.03.003

58. Stoppini L, Buchs PA, Muller D. A simple method for organotypic cultures of nervous tissue. J Neurosci Methods. 1991;37(2):173-182. doi:10.1016/0165-0270(91)90128-M

59. Mewes A, Franke H, Singer D. Organotypic brain slice cultures of adult transgenic P301S mice-a model for tauopathy studies. PLoS One. 2012;7(9):e45017. doi:10.1371/journal.pone.0045017

60. Adamski V, Schmitt C, Ceynowa F, et al. Effects of sequentially applied single and combined temozolomide, hydroxychloroquine and AT101 treatment in a long-term stimulation glioblastoma in vitro model. J Cancer Res Clin Oncol. 2018;144(8):1475-1485. doi:10.1007/s00432-018-2680-y

61. Lin MS, Lee YH, Chiu WT, Hung KS. Curcumin provides neuroprotection after spinal cord injury. J Surg Res. 2011;166(2):280-289. doi:10.1016/j.jss.2009.07.001

62. Zhang N, Wei G, Ye J, et al. Effect of curcumin on acute spinal cord injury in mice via inhibition of inflammation and TAK1 pathway. Pharmacol Rep. 2017;69(5):1001-1006. doi:10.1016/j.pharep.2017.02.012

63. Nelson KM, Dahlin JL, Bisson J, Graham J, Pauli GF, Walters MA. The essential medicinal chemistry of curcumin. J Med Chem. 2017;60(5):1620-1637. doi:10.1021/acs.jmedchem.6b00975

64. Potter KA, Jorfi M, Householder KT, Foster EJ, Weder C, Capadona JR. Curcumin-releasing mechanically adaptive intracortical implants improve the proximal neuronal density and blood-brain barrier stability. Acta Biomater. 2014;10(5):2209-2222. doi:10.1016/ j.actbio.2014.01.018

65. Raza F, Zafar H, You X, Khan A, Wu J, Ge L. Cancer nanomedicine: focus on recent developments and self-assembled peptide nanocarriers. J Mater Chem B. 2019;7(48):7639-7655. doi:10.1039/C9TB01842E

66. Roy B, Guha P, Bhattarai R, et al. Influence of lipid composition, $\mathrm{pH}$, and temperature on physicochemical properties of liposomes with curcumin as model drug. J Oleo Sci. 2016;65(5):399-411. doi:10.5650/jos.ess 15229

67. Franze S, Selmin F, Samaritani E, Minghetti P, Cilurzo F. Lyophilization of liposomal formulations: still necessary, still challenging. Pharmaceutics. 2018;10:3. doi:10.3390/pharmaceutics 10030139

68. van Winden ECA, Crommelin DJ. Long term stability of freeze-dried, lyoprotected doxorubicin liposomes. Eur J Pharm Biopharm. 1997;43 (3):295-307. doi:10.1016/S0939-6411(97)00058-1 
69. Mohammed AR, Coombes AG, Perrie Y. Amino acids as cryoprotectants for liposomal delivery systems. Eur J Pharm Sci. 2007;30 (5):406-413. doi:10.1016/j.ejps.2007.01.001

70. Zhao M, Zhao M, Fu C, Yu Y, Fu A. Targeted therapy of intracranial glioma model mice with curcumin nanoliposomes. Int J Nanomedicine. 2018;13:1601-1610.

71. Sofroniew MV. Molecular dissection of reactive astrogliosis and glial scar formation. Trends Neurosci. 2009;32(12):638-647. doi:10.1016/ j.tins.2009.08.002

72. Jin CY, Lee JD, Park C, Choi YH, Kim GY. Curcumin attenuates the release of pro-inflammatory cytokines in lipopolysaccharide-stimulated BV2 microglia. Acta Pharmacol Sin. 2007;28(10):1645-1651. doi:10. 1111/j.1745-7254.2007.00651.x

73. Karlstetter M, Lippe E, Walczak Y, et al. Curcumin is a potent modulator of microglial gene expression and migration. J Neuroinflammation. 2011;8(1):125. doi:10.1186/1742-2094-8-125

74. Guo L, Xing Y, Pan R, et al. Curcumin protects microglia and primary rat cortical neurons against HIV-1 gp120-mediated inflammation and apoptosis. PLoS One. 2013;8(8):e70565. doi:10.1371/ journal.pone.0070565

75. Cianciulli A, Calvello R, Porro C, Trotta T, Salvatore R, Panaro MA. $\mathrm{PI} 3 \mathrm{k} / \mathrm{Akt}$ signalling pathway plays a crucial role in the anti-inflammatory effects of curcumin in LPS-activated microglia. Int Immunopharmacol. 2016;36:282-290. doi:10.1016/j.intimp.2016. 05.007

76. Ding F, Li F, Li Y, et al. HSP60 mediates the neuroprotective effects of curcumin by suppressing microglial activation. Exp Ther Med. 2016;12(2):823-828. doi:10.3892/etm.2016.3413

77. Qin X, Qiao H, Wu S, Cheng J, Wan Q, Liu R. Curcumin inhibits monocyte chemoattractant Protein-1 expression in TNF-alpha induced astrocytes through AMPK pathway. Neurochem Res. 2018;43(4):775-784. doi:10.1007/s11064-018-2479-x

78. Seyedzadeh MH, Safari Z, Zare A, et al. Study of curcumin immunomodulatory effects on reactive astrocyte cell function Int Immunopharmacol. 2014;22(1):230-235. doi:10.1016/j.intimp. 2014.06.035

79. Daverey A, Agrawal SK. Curcumin alleviates oxidative stress and mitochondrial dysfunction in astrocytes. Neuroscience. 2016;333:92-103. doi:10.1016/j.neuroscience.2016.07.012
80. Kolter M, Wittmann M, Koll-Weber M, Suss R. The suitability of liposomes for the delivery of hydrophobic drugs - a case study with curcumin. Eur J Pharm Biopharm. 2019;140:20-28. doi:10.1016/j. ejpb.2019.04.013

81. Bollimpelli VS, Kumar P, Kumari S, Kondapi AK. Neuroprotective effect of curcumin-loaded lactoferrin nano particles against rotenone induced neurotoxicity. Neurochem Int. 2016;95:37-45. doi:10.1016/j. neuint.2016.01.006

82. Krupa P, Svobodova B, Dubisova J, Kubinova S, Jendelova P, Machova Urdzikova L. Nano-formulated curcumin (Lipodisq) modulates the local inflammatory response, reduces glial scar and preserves the white matter after spinal cord injury in rats. Neuropharmacology. 2019;155:54-64. doi:10.1016/j.neuropharm.2019.05.018

83. Naeimi R, Safarpour F, Hashemian M, et al. Curcumin-loaded nanoparticles ameliorate glial activation and improve myelin repair in lysolecithin-induced focal demyelination model of rat corpus callosum. Neurosci Lett. 2018;674:1-10. doi:10.1016/j.neulet.2018.03.018

84. Hoppe JB, Haag M, Whalley BJ, Salbego CG, Cimarosti H. Curcumin protects organotypic hippocampal slice cultures from Abeta1-42-induced synaptic toxicity. Toxicol in Vitro. 2013;27 (8):2325-2330. doi:10.1016/j.tiv.2013.10.002

85. Hoppe JB, Frozza RL, Pires EN, Meneghetti AB, Salbego C. The curry spice curcumin attenuates beta-amyloid-induced toxicity through beta-catenin and PI3K signaling in rat organotypic hippocampal slice culture. Neurol Res. 2013;35(8):857-866. doi:10.1179/ 1743132813Y.0000000225

86. Choi GY, Kim HB, Hwang ES, et al. Curcumin alters neural plasticity and viability of intact hippocampal circuits and attenuates behavioral despair and COX-2 expression in chronically stressed rats. Mediators Inflamm. 2017;2017:6280925. doi:10.1155/2017/6280925

87. Dal Ben M, Bottin C, Zanconati F, Tiribelli C, Gazzin S. Evaluation of region selective bilirubin-induced brain damage as a basis for a pharmacological treatment. Sci Rep. 2017;7:41032. doi:10.1038/ srep41032

88. Drion CM, Kooijman L, Aronica E, et al. Curcumin reduces development of seizurelike events in the hippocampal-entorhinal cortex slice culture model for epileptogenesis. Epilepsia. 2019;60 (4):605-614. doi:10.1111/epi.14667
International Journal of Nanomedicine

\section{Publish your work in this journal}

The International Journal of Nanomedicine is an international, peerreviewed journal focusing on the application of nanotechnology in diagnostics, therapeutics, and drug delivery systems throughout the biomedical field. This journal is indexed on PubMed Central, MedLine, CAS, SciSearch ${ }^{\circledR}$, Current Contents ${ }^{\circledR} /$ Clinical Medicine,
Journal Citation Reports/Science Edition, EMBase, Scopus and the Elsevier Bibliographic databases. The manuscript management system is completely online and includes a very quick and fair peer-review system, which is all easy to use. Visit http://www.dovepress.com/ testimonials.php to read real quotes from published authors. 